\title{
Spectral energy distributions of a large sample of BL Lacertae objects ${ }^{\star}$
}

\author{
E. Nieppola ${ }^{1}$, M. Tornikoski ${ }^{2}$, and E. Valtaoja ${ }^{1,3}$ \\ 1 Tuorla Observatory, Väisäläntie 20, 21500 Piikkiö, Finland \\ e-mail: eni@kurp.hut.fi \\ 2 Metsähovi Radio Observatory, Metsähovintie 114, 02540 Kylmälä, Finland \\ 3 Dept. of Physical Sciences, University of Turku, 20100 Turku, Finland
}

Received 26 April 2005 / Accepted 26 August 2005

\section{ABSTRACT}

\begin{abstract}
We have collected a large amount of multifrequency data for objects in the Metsähovi Radio Observatory BL Lacertae sample and computed their spectral energy distributions (SED) in the $\log v-\log v F-$ representation. This is the first time the SEDs of BL Lacs have been studied with a sample of over 300 objects. The synchrotron components of the SEDs were fitted with a parabolic function to determine the synchrotron peak frequency, $v_{\text {peak }}$. We checked the dependence between luminosities at several frequency bands and synchrotron peak frequency to test the blazar sequence scenario, which states that the source luminosity depends on the location of the synchrotron peak. We also calculated broad band spectral indices and plotted them against each other and $v_{\text {peak }}$.

The range of $v_{\text {peak }}$ in our study was considerably extended compared to previous studies. There were 22 objects for which $\log v_{\text {peak }}>19$. The data shows that at $5 \mathrm{GHz}, 37 \mathrm{GHz}$, and $5500 \AA$, there is negative correlation between luminosity and $v_{\text {peak }}$, whereas in X-rays the correlation turns slightly positive. There is no significant correlation between source luminosity at synchrotron peak and $v_{\text {peak }}$. Several low radio luminosity-low energy peaked BL Lacs were found. The negative correlation between broad band spectral indices and $v_{\text {peak }}$ is also significant, although there is substantial scatter. Therefore we find that neither $\alpha_{\mathrm{rx}}$ nor $\alpha_{\mathrm{ro}}$ can be used to determine the synchrotron peak of BL Lacs. On the grounds of our results, we conclude that the blazar sequence scenario is not valid. In all our results, the BL Lac population is continuous with no hint of the bimodality of the first BL Lac samples.
\end{abstract}

Key words. galaxies: active - BL Lacertae objects: general - radiation mechanisms: non-thermal

\section{Introduction}

BL Lacertae (BL Lacs) are a subclass of active galactic nuclei (AGN). They are characterized by the lack of strong emission lines, rapid variability at all wavelengths, and strong polarization. Their spectral energy distribution (SED), in the $\log v$ - $\log v F$-representation, consists of a synchrotron component at lower frequencies and an inverse Compton component at higher frequencies. The peculiar traits of the BL Lac class are most likely caused by Doppler-boosted radiation emanating from a relativistic jet aligned close to the line of sight (Urry \& Padovani 1995).

Traditionally, BL Lacs have been discovered in either radio or X-ray band, which led to their classification as radioselected (RBL) and X-ray-selected (XBL) BL Lacs. Bestknown RBL samples include the 1 Jy (Stickel et al. 1991), S4 (Stickel \& Kühr 1994), and S5 (Kühr \& Schmidt 1990) samples, and among the most important XBL samples are the

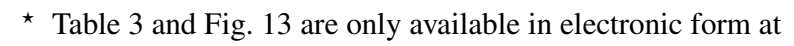
http://www.edpsciences.org
EMSS (Gioia et al. 1990; Stocke et al. 1991) and Slew Survey (Perlman et al. 1996).

The two classes have different properties: RBLs are more variable, are more luminous at radio and optical wavelengths, and have a higher polarization (Stocke et al. 1985; Jannuzi et al. 1994). XBLs have a higher starlight fraction, 30-50\% (Stocke et al. 1985), and their morphology is less core-dominated in the radio than that of RBLs (Perlman \& Stocke 1993). Due to these differences, they were initially regarded as separate classes of AGN. However, in recent years samples including intermediate objects have been found in surveys which combine X-ray and radio observations. These include the RGB (RASS-Green Bank) sample (Laurent-Muehleisen et al. 1999), the Deep X-Ray Radio Blazar Survey (DXRBS) (Perlman et al. 1998; Landt et al. 2001), and the REX Survey (Caccianiga et al. 1999). Their discovery has strengthened the view that the BL Lac population is continuous and that RBLs and XBLs represent the opposite ends of the continuum. The reason for such a continuity lies in the cutoff frequency of the synchrotron component in the SED. The synchrotron peak frequency of RBLs is in the radio/IR band, and for XBLs the peak is mostly in 
the UV/X-ray band (Giommi et al. 1995). The intermediate BL Lacs (IBL) have their synchrotron peak in the optical wavelengths. This explains why they were not observed in the first surveys. Following the synchrotron cutoff model, the terminology came to describe the physical difference of the two classes: the RBL/XBL-division was replaced by division into low-energy-peaked BL Lacs (LBL) and high-energy-peaked BL Lacs (HBL) (Padovani \& Giommi 1995). Most RBLs are LBLs and most XBLs are HBLs, but not all. The class boundaries can be loosely defined as $v_{\text {peak }} \approx 10^{13-14} \mathrm{~Hz}$ for LBLs, $v_{\text {peak }} \approx 10^{15-16} \mathrm{~Hz}$ for IBLs, and $v_{\text {peak }} \approx 10^{17-18} \mathrm{~Hz}$ for HBLs.

Fossati et al. (1998) linked the shape of the SED and the synchrotron peak frequency to the source luminosity: the lower the peak frequency, the more luminous the source. This would mean that LBLs are intrinsically more luminous than HBLs. This sequencing is based on the absence of highluminosity HBLs and low-luminosity LBLs. However, recently Giommi et al. (2005) reported the possible discovery of highluminosity HBLs in the Sedentary Survey, and evidence of lowpower LBLs has also been discovered (Padovani et al. 2003; Caccianiga \& Marchã 2004). These findings are at odds with the trend presented by Fossati et al.

Ghisellini (1999) suggested that there is a class of BL Lacs whose synchrotron peak lies at even higher frequencies than that of conventional HBLs, $v_{\text {peak }}>10^{19} \mathrm{~Hz}$. These objects can be called ultra-high-energy synchrotron peak BL Lacs (UHBLs) (Giommi et al. 2001). Following the dependency of the SED shape and luminosity, UHBLs are thought to be extremely faint at radio wavelengths, which is why they have escaped notice. Extensive $\gamma$-ray observations are needed to unambiguously identify them.

In this paper we have taken a new approach to studying the properties of the BL Lac population. Our goal is to plot the SEDs of the Metsähovi Radio Observatory BL Lac sample, which comprises nearly 400 objects, including objects from all the best-known surveys at radio and X-ray wavelengths. This way we can examine the population properties of a sample with a wide range of attributes, instead of focusing on one or two limited surveys.

The aim of this paper was to test both the continuity of the BL Lac population and the blazar sequence scenario and to assign a SED-based classification to those objects that previously had none. A large database of flux measurements has been collected and SEDs are plotted for more than 300 BL Lacs. Each object is classified as LBL, IBL, or HBL. The relationship of synchrotron peak frequency and luminosities at several frequencies was also tested along with the properties of broad band spectral indices. Throughout this paper, we assume $H_{0}=65 \mathrm{~km} \mathrm{~s}^{-1} \mathrm{Mpc}^{-1}$ and $\Omega_{0}=1$. All statistical tests were carried out using Unistat 5.5 software.

\section{The sample}

The Metsähovi BL Lac sample includes 381 objects selected from the Veron-Cetty \& Veron BL Lac Catalogue (Veron-Cetty \& Veron 2000), hereafter VCV2000, and 17 objects from the literature. Given the northern location of the Metsähovi observatory, the source with the lowest declination in the sample is
PKS 2223-114 at $\delta=-11: 13: 41$. The list of sample sources (Table 3 ) is published electronically. A large part of the objects in VCV2000 are from well-known and well-defined samples such as the 1 Jy, S4, S5, EMSS, and the Einstein Slew Survey. The BL Lacs from the first release of DXRBS identifications (Perlman et al. 1998) are also included. No selection criteria (other than declination), in addition to the ones in the original surveys, were imposed on the sample. The aim was to examine the behaviour of an extensive sample containing all known BL Lacs up to the year 2000.

According to VCV2000, the Metsähovi BL Lac sample can be further classified as follows: $63 \%$ are confirmed, $3 \%$ probable and $8 \%$ possible BL Lacs, $14 \%$ are objects with high optical polarization, and $12 \%$ (including the BL Lacs taken from literature) lack any subclassification. In the sample there are 6 sources that are not in the later editions of the Veron-Cetty \& Veron BL Lac Catalogues (Veron-Cetty \& Veron 2001, 2003). These objects were excluded from the data analysis performed in this paper.

\section{The data}

In order to plot as accurate SEDs as possible, a large amount of data from several wavelengths was collected. Because simultaneous multifrequency flux measurements are not available, datapoints from different epochs were searched from databases and the literature.

\subsection{Radio data}

The starting point in collecting radio data were the Metsähovi observations at $37 \mathrm{GHz}$ from late 2001 to January 2004. The full data set and a more detailed analysis of the $37 \mathrm{GHz}$ behaviour of the sample sources will be published in a forthcoming paper (Nieppola et al., in preparation for A\&A). Of the BL Lac sample, 137 objects were detected at $\sigma \geq 4$. There were $255 \mathrm{BL}$ Lacs that were not detected and 6 that had not yet been observed during the time mentioned. The limiting flux of the Metsähovi radio telescope is about $0.2 \mathrm{Jy}$ under optimal weather conditions.

Our group also obtained flux density data at higher radio frequencies from our observations with the SwedishESO Submillimetre Telescope (SEST) between 1987 and 2003 at $3 \mathrm{~mm}$ and $1.3 \mathrm{~mm}$. For some objects we got data from the RATAN observatory at frequencies $2.3,4.8,7.7,11.2$, 21.8, and $30 \mathrm{GHz}$ (Tornikoski et al., in preparation for A\&A). Additional low frequency datapoints were found in VCV2000 and the WGA-catalogue (White et al. 1996).

The large majority of radio data were obtained from the Astrophysical Catalogues Support System (CATS) maintained by the Special Astrophysical Observatory, Russia ${ }^{1}$. The search results from CATS contained data from over 140 different catalogues and more than 100 radio frequencies.

\footnotetext{
${ }^{1}$ http://cats.sao.ru/
} 


\subsection{Data from other frequency bands}

The IR datapoints are from CATS, originating from the IRASand 2MASS-catalogues. The wavelengths used are 1.25, 1.65, $2.0,2.17,12,25,60$ and $100 \mu \mathrm{m}$. The optical data also are mainly from CATS. Some datapoints from $V$-band were added from Donato et al. (2001).

The X-ray data are from Einstein- and ROSAT-catalogues. The majority is from WGACAT (White et al. 1996) and the RBSC-catalogue (Voges et al. 1999). Datapoints were also collected from the following papers: Donato et al. (2001), Lamer et al. (1996), and Laurent-Muehleisen et al. (1999). EMSS-data were included as well (Gioia et al. 1990; Stocke et al. 1991). All of the data from gamma region came from the Third EGRET Catalogue (Hartman et al. 1999). Such data were available for only 14 objects.

\section{Computing the SEDs}

For all sources with a sufficient number of datapoints, a SED was plotted in the form $\log v-\log v F$. All frequencies used are observed frequencies and they have not been reduced to rest frame frequencies. The synchrotron component of the SED was fitted with a parabolic function

$y=A x^{2}+B x+C$

in order to determine the synchrotron peak frequency $v_{\text {peak }}=$ $-B / 2 A$. The fitting was successful for 308 objects in total: the rest were too sparsely sampled. Among the fitted sources there were 4 which do not appear in the later versions of the Veron-Cetty \& Veron catalogues of BL Lacertae objects, as mentioned in Sect. 2. Therefore they are not included in the analyses and discussion in this paper hereafter. The decision whether or not to include X-ray datapoints in the fit was based solely on a visual estimate for each individual object. All the SEDs (Fig. 13) are published electronically. The synchrotron peak frequencies are shown in Table 3 Col. 5 .

We note that using a simple parabolic function in the fitting produces some error, especially among HBLs. In their case the X-ray datapoints are typically included in the rising synchrotron component, and therefore the parabola peaks after the $\mathrm{X}$-ray domain. In reality, the synchrotron peak is expected to occur at or very close to the X-ray wavelengths and the decline to be more rapid. Thus the peak frequencies of the most extreme objects can be exaggerated.

The objects were assigned an LBL/IBL/HBL classification according to $v_{\text {peak }}$. On the basis of the criteria of Padovani \& Giommi (1995), we decided to draw the boundaries as follows: for LBLs, $\log v_{\text {peak }}<14.5$, for IBLs $14.5<\log v_{\text {peak }}<16.5$, and for HBLs $\log v_{\text {peak }}>16.5$. Thus the peak frequencies of LBLs stretch up to the optical region, IBLs peak in the optical and UV-bands and HBLs from soft X-rays upwards.

\subsection{The distribution of $v_{\text {peak }}$}

When the objects were classified as LBL/IBL/HBL according to their $v_{\text {peak }}$, as described in the previous section, the division resulted in the three classes being almost equal in size. There

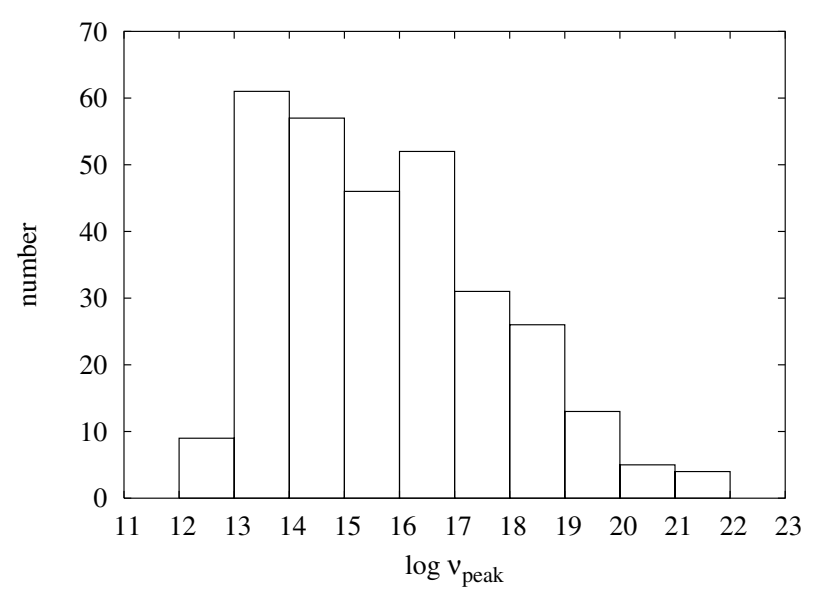

Fig. 1. The distribution of the synchrotron peak frequencies in the Metsähovi sample.

Table 1. Division of observational BL Lac classes to physical ones.

\begin{tabular}{c|ccc}
\hline \hline & LBL & IBL & HBL \\
\hline RBL & $84 \%$ & $10 \%$ & $6 \%$ \\
IBL & $22 \%$ & $36 \%$ & $42 \%$ \\
XBL & $21 \%$ & $25 \%$ & $54 \%$ \\
\hline
\end{tabular}

were 98 LBLs, 96 IBLs, and 110 HBLs. The distribution is smooth and decreases steadily towards the higher peak frequencies (Fig. 1). This can be a real effect, suggesting that sources in which electrons are accelerated to extreme energies are intrinsically rare or are due to selection effects in surveys. The frequency interval $v_{\text {peak }}=10^{13-14} \mathrm{~Hz}$ is the most populated.

Table 1 presents how the classification with respect to the observational band relates to the one based on the SED. Here RBL classification has been assigned to objects in the $1 \mathrm{Jy}$ and S4 surveys, IBL to objects in RGB and $200 \mathrm{mJy} \mathrm{BL} \mathrm{Lac}$ (Bondi et al. 2001) surveys, and XBL to objects in the Einstein Slew Survey. This RBL/XBL classification has been adopted from Giommi et al. (1995). Among the 304 sources for which the SED could be plotted, there were 31 RBLs, 115 IBLs, and 48 XBLs. Several objects got multiple classifications.

Table 1 clearly demonstrates how surveys in the X-ray energies are more prone to finding low-energy BL Lacs than radio surveys are to finding HBLs. A large majority of RBLs really are LBLs. In fact, the $6 \%$ of RBLs that turned out to be HBLs are the radio-luminous Mrk 421 and Mrk 501. Objects in the RGB and 200 mJy samples are more likely to be HBLs than LBLs, and only a third of them are truly intermediate. Of the XBLs, only half are HBLs and over $20 \%$ are actually lowenergy BL Lacs. These figures are certainly affected by the arbitrariness of the dividing boundaries between the classes, but the overall trend is expected to remain. It results from the fact that the X-ray luminosities of the samples in question are roughly the same, whereas radio luminosities differ greatly. 
Table 2. Objects for which $\log v_{\text {peak }}>19$.

\begin{tabular}{lccc}
\hline \hline Source & RA $(\mathrm{J} 2000)$ & Dec $(\mathrm{J} 2000)$ & $\log v_{\text {peak }}$ \\
\hline 1ES 0229+200 & $02: 32: 48.6$ & $+20: 17: 17$ & 19.45 \\
RXS J0314.3+0620 & $03: 14: 23.9$ & $+06: 19: 57$ & 19.57 \\
2E 0323+0214 & $03: 26: 13.9$ & $+02: 25: 14$ & 19.87 \\
2E 0414+0057 & $04: 16: 52.4$ & $+01: 05: 24$ & 20.71 \\
1ES 0502+675 & $05: 07: 56.1$ & $+67: 37: 24$ & 19.18 \\
EXO 0706.1+5913 & $07: 10: 30.1$ & $+59: 08: 21$ & 21.05 \\
RXS J0847.2+1133 & $08: 47: 12.9$ & $+11: 33: 52$ & 19.13 \\
1ES 0927+500 & $09: 30: 37.5$ & $+49: 50: 25$ & 21.13 \\
RXS J1008.1+4705 & $10: 08: 11.3$ & $+47: 05: 20$ & 19.67 \\
RXS J1012.7+4229 & $10: 12: 44.3$ & $+42: 29: 57$ & 20.97 \\
EXO 1449.9+2455 & $11: 49: 30.3$ & $+24: 39: 27$ & 19.83 \\
PG 1218+304 & $12: 21: 21.9$ & $+30: 10: 37$ & 19.14 \\
RXS J1319.5+1405 & $13: 19: 31.7$ & $+14: 05: 34$ & 20.85 \\
RXS J1341.0+3959 & $13: 41: 05$ & $+39: 59: 45$ & 20.06 \\
RXS J1353.4+5601 & $13: 53: 28$ & $+56: 00: 55$ & 19.23 \\
RXS J1410.5+6100 & $14: 10: 31.7$ & $+61: 00: 10$ & 20.25 \\
2E 1415+2557 & $14: 17: 56.6$ & $+25: 43: 25$ & 19.24 \\
RXS J1456.0+5048 & $14: 56: 03.7$ & $+50: 48: 25$ & 19.94 \\
RXS J1458.4+4832 & $14: 58: 28$ & $+48: 32: 40$ & 21.46 \\
1ES 1533+535 & $15: 35: 00.8$ & $+53: 20: 37$ & 19.68 \\
RXS J1756.2+5522 & $17: 56: 15.9$ & $+55: 22: 18$ & 19.90 \\
RXS J2304.6+3705 & $23: 04: 36.6$ & $+37: 05: 08$ & 21.01 \\
\hline
\end{tabular}

\subsection{UHBL candidates}

As Fig. 1 shows, there were several objects in the sample whose synchrotron peak frequency was extremely high. Usually objects with $\log v_{\text {peak }}>17$ are considered as extreme; here the number of such objects was 80 , approximately $26 \%$ of all the fits. For 22 objects $\log v_{\text {peak }}>19$ (Table 2) and for 9 objects even $\log v_{\text {peak }}>20$, corresponding to a peak energy of $\sim 0.4 \mathrm{MeV}$. The SEDs of these sources were, on the whole, very sparsely sampled, typically with datapoints from radio, optical, and X-ray bands, and should be treated with caution. That said, we note that even for objects with $\log v_{\text {peak }}>20$, the datapoints fit very well on the rising parabolic function. We note again that the actual position of the peak is probably exaggerated by the use of a parabolic fitting function, as mentioned in Sect. 4, meaning the peak frequencies of these objects cannot be considered as definite. Because they peak near the $\mathrm{MeV}$-region, these sources would be excellent candidates for $\gamma$-observations.

\section{Correlation between $v_{\text {peak }}$ and luminosity}

\subsection{High radio frequency $(37 \mathrm{GHz})$}

Out of the three BL Lac classes, LBLs had $37 \mathrm{GHz}$ detections for $81 \%$ of the sources, IBLs for $36 \%$, and HBLs for $12 \%$. This seems to indicate that most HBLs have a radio flux well below the flux limit of the Metsähovi telescope. This prompted

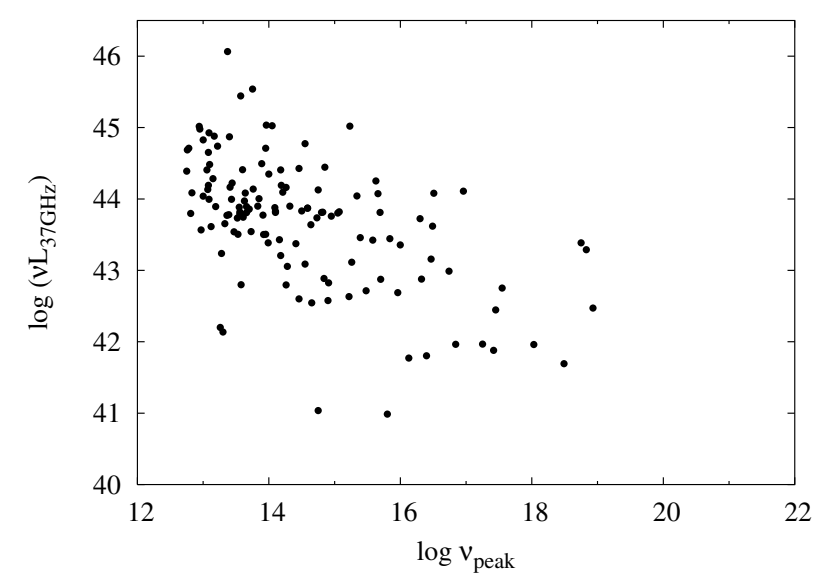

Fig. 2. Radio luminosity at $37 \mathrm{GHz}$ plotted against synchrotron peak frequency.

us to study the possible correlation between synchrotron peak frequency and $37 \mathrm{GHz}$ source luminosity more closely.

To calculate the luminosities, redshift data was collected from VCV2000, Stocke et al. (1991), Laurent-Muehleisen et al. (1999), Donato et al. (2001), and the SIMBAD database ${ }^{2}$. For sources with no redshift available, we used $z=0.4$. Both a detection at $37 \mathrm{GHz}$ and synchrotron peak frequency $v_{\text {peak }}$ were available for 132 objects.

\footnotetext{
${ }^{2}$ http://simbad.u-strasbg.fr
} 


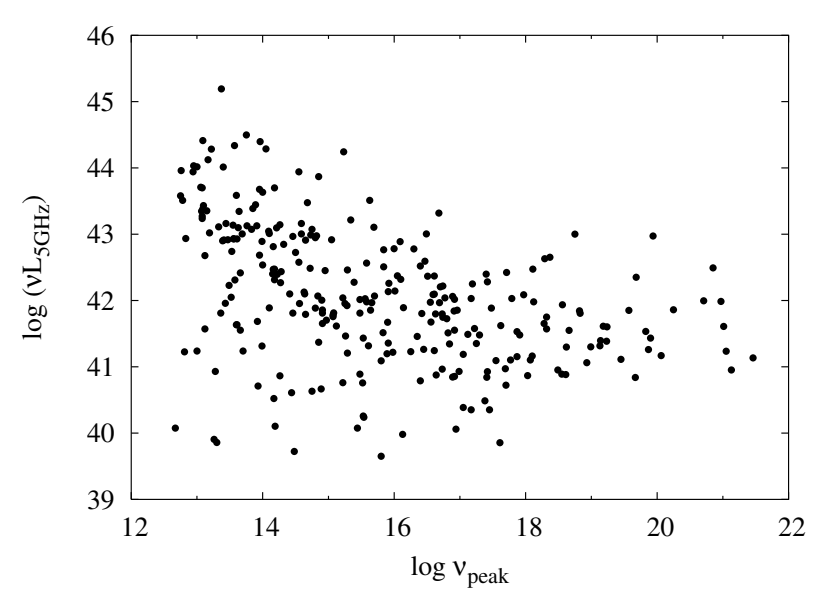

Fig. 3. Radio luminosity at $5 \mathrm{GHz}$ plotted against synchrotron peak frequency.

When the $37 \mathrm{GHz}$ luminosity is plotted against the synchrotron peak frequency (Fig. 2), the correlation is easily seen. LBLs are at the high-luminosity end of the plot, and the luminosity drops towards the high-energy regime. According to the Spearman Rank Correlation Test, there is significant negative correlation at the $99 \%$ confidence level.

No real evidence of a population of low-luminosity LBLs or high-luminosity HBLs was found. However, there are two LBLs with luminosities that are almost as low as those of HBLs, disrupting the declining trend. In addition, the lowest luminosities are not claimed by objects at the high end of the peak frequency range, but by two IBLs with $\log v_{\text {peak }}<16$. The radio luminosities are widely scattered. An object with $\log v L=44$ can have peak frequency values ranging from $\log v_{\text {peak }}=13$ to $\log v_{\text {peak }}=17$, approximately. Thus the radio luminosity cannot be used to determine the peak frequency of the source.

\subsection{Low radio frequency $(5 \mathrm{GHz})$}

The small number of HBL datapoints at $37 \mathrm{GHz}$ convinced us to also test the correlation at $5 \mathrm{GHz}$. The number of available datapoints rose to 280, and the correlation plot changed drastically (Fig. 3). The most noticeable difference is the appearance of several low-luminosity LBLs (lower left of the figure). They even reach lower luminosities than any of the HBLs. The overall negative correlation is still present and significant at a 99\% level. This differs from the result obtained by Padovani et al. (2003). However, their DXRBS BL Lac sample consisted of only 31 objects that mainly represent the LBL/IBL end of the plot. This is the region with the most scatter in our figure, and within this limited area, the correlation is less obvious even with a larger number of datapoints. Only when the whole range of values of $\log v_{\text {peak }}$ is considered does the trend become evident.

When compared with the corresponding figure of Fossati et al. (1998), our figure has much more scatter. A part of it is caused by a larger number of datapoints, but on closer examination, our $\log v_{\text {peak }}$ values for the Slew Survey are spread much more widely. In Fossati et al. (1998), the Slew Survey

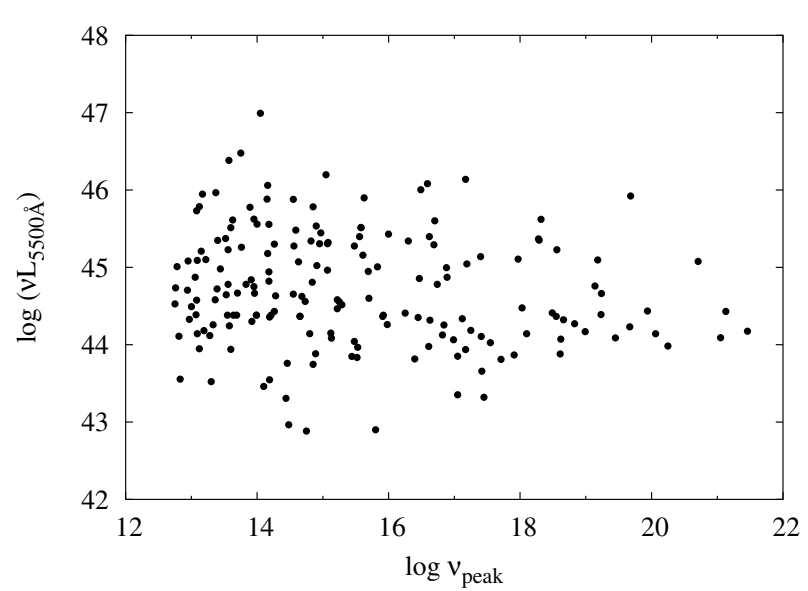

Fig. 4. Optical luminosity at $5500 \AA$ plotted against synchrotron peak frequency.

objects have $\log v_{\text {peak }}=15-19$, while in our study they have $\log v_{\text {peak }} \approx 13-21$. The $1 \mathrm{Jy}-$ sample takes on similar peak frequency values in both studies.

We note that there is no evidence of very high-luminosity HBLs. In fact, the extreme HBLs adopt quite intermediate luminosity values also avoiding the low-luminosity region. However, the spectra of possible high-luminosity HBLs would very likely be totally featureless because of the powerful nucleus, and thus the object would lack a redshift estimation. Therefore the redshift value assigned to featureless sources could have a big impact on the luminosity correlations and the appearance of high-luminosity HBLs (see Sect. 5.6).

\subsection{Optical region $(5500 \AA)$}

Figure 4 shows the optical luminosity at wavelength $5500 \AA$ plotted against $\log v_{\text {peak }}$. There is a significant, slightly negative correlation present at a $95 \%$ level. We see again that LBLs have more scatter than HBLs. Overall, the correlation is much less evident than in the case of radio luminosity.

\subsection{X-ray region}

In Fig. 5 we have plotted the correlation between X-ray luminosity and $\log v_{\text {peak }}$. In calculating the luminosities we used both $1 \mathrm{keV}$ data and data from the ROSAT band (0.1-2.4 keV). The error produced by the bandwidth difference is negligible when only the statistical properties of a large sample are considered. We note that in the case of X-ray luminosity, the correlation is positive and significant at a $99 \%$ level. This contrasts with the blazar sequence scenario. Fossati et al. (1998) state that the overall luminosity of HBLs is lower than that of LBLs (see their Fig. 12). While they admit that in the X-ray band objects exhibit complex behaviour, the systematic rising trend presented by our findings is not predicted.

\subsection{Peak luminosity $L_{\text {peak }}$}

In addition to luminosities at defined frequency bands, we calculated the luminosity at the synchrotron peak frequency for 


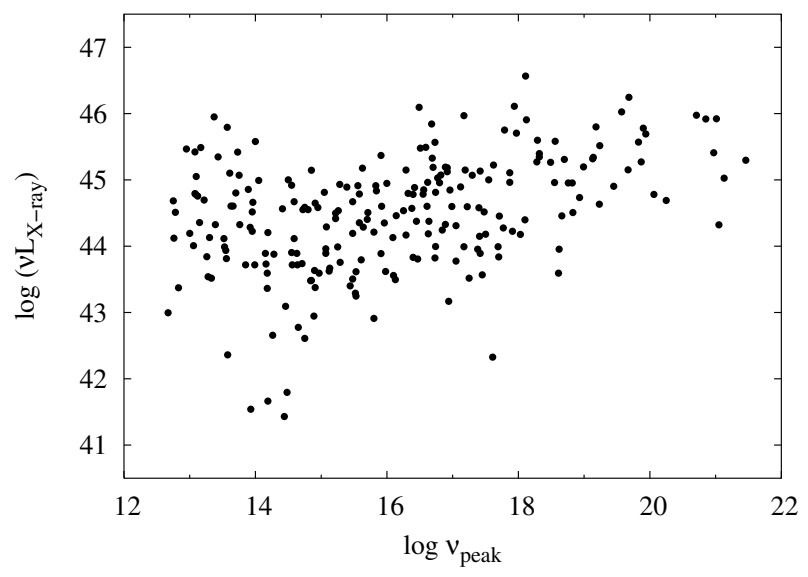

Fig. 5. X-ray luminosity at $1 \mathrm{keV}$ and ROSAT band plotted against synchrotron peak frequency.

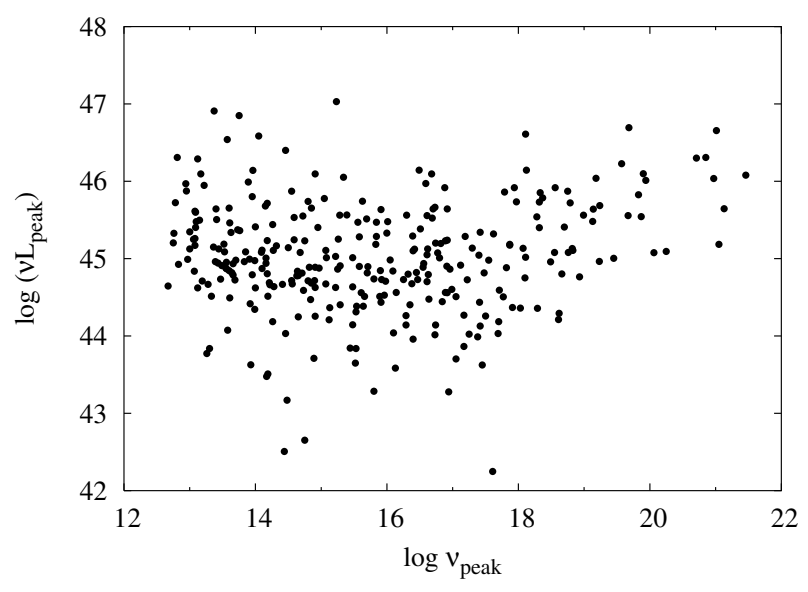

Fig. 6. Luminosity at the synchrotron peak frequency plotted against synchrotron peak frequency.

each source. This is plotted against $\log v_{\text {peak }}$ in Fig. 6. There is no significant correlation, so that we can decisively say that the source luminosity does not depend on the synchrotron peak frequency. Figure 7 of Fossati et al. (1998) also shows the dependence of $L_{\text {peak }}$ and $\log v_{\text {peak }}$. In their study there is a significant, yet weak correlation. Again, we notice that a larger number of datapoints and a wider range of $\log v_{\text {peak }}$ reveal the true behaviour of the population and the lack of correlation. If only objects with $\log v_{\text {peak }}<17$ are considered, there is a weak negative correlation in our sample also. On the other hand, when the high-energy tail with $\log v_{\text {peak }}>17$ is tested, we find a significant positive correlation. Therefore the distribution almost seems to assume a concave shape.

\subsection{The effect of $z$ on luminosity correlations}

Giommi et al. (2005) found numerous candidates for highluminosity HBLs in the Sedentary Survey. All these objects seemed to reside at high redshifts $(z \geq 0.7)$. In our luminosity calculations we assumed $z=0.4$ for featureless objects, which is a low value compared to Giommi et al. Using too low a redshift value for a significant part of the sources would lead to a serious underestimation of luminosities. To take this into

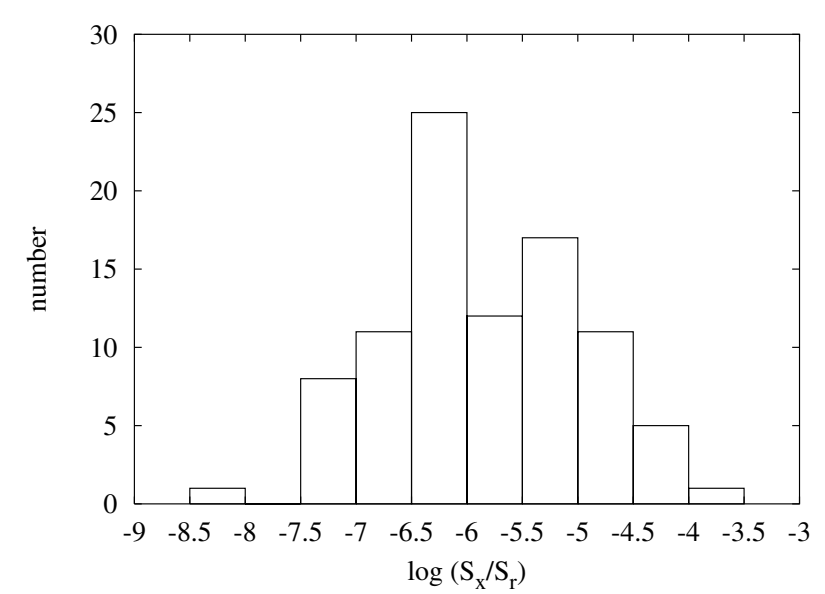

Fig. 7. The distribution of $\log \left(S_{x} / S_{r}\right)$.

account, we also tested the luminosity correlations by assigning redshifts $z=0.7, z=1$, and $z=1.5$ to those objects that had none.

The effect was most pronounced in the case of radio luminosities at 5 and $37 \mathrm{GHz}$. The number of relatively highluminosity HBLs increased with the higher assumed redshift. However, only at very high reshift values $(z=1$ or $z=1.5)$ did the luminosities of a few HBLs become roughly comparable to those of LBLs. Considering the population average, $z_{\mathrm{av}}=0.33$, it is questionable whether all featureless sources would have $z \geq 1$, although for some of them this may be the case. Therefore, while the existence of high-luminosity HBLs in this sample is possible, we do not expect the correlations to be affected by them.

In other frequency bands than radio, the effect of an increasing redshift was negligible. The shape of the correlations did not change notably, while the scatter increased somewhat. We note that in all wavelengths and for all assumed redshift values, the significance of the statistical correlations remained the same.

\section{Other properties of the sample}

\section{1. $\log \left(S_{x} / S_{r}\right)$-distribution}

The $\log \left(S_{x} / S_{r}\right)$-distribution has traditionally been used to point out the bimodality in the BL Lac population. The dividing line between RBLs and XBLs has been $\log \left(S_{x} / S_{r}\right)=-5.5$ (Laurent-Muehleisen et al. 1999), when the fluxes are in the same units and X-ray and radio frequencies are $0.1-2.4 \mathrm{keV}$ and $5 \mathrm{GHz}$, respectively. Here we calculated the distribution to check the assumed continuity of the sample. X-ray fluxes are from the ROSAT band $0.1-2.4 \mathrm{keV}$, and radio measurements are from Metsähovi at $37 \mathrm{GHz}$. If there was more than one flux measurement from one frequency, the average value was used.

The distribution is indeed unbroken with an average of $\log \left(S_{x} / S_{r}\right)=-5.85$ (Fig. 7). The most populated interval is $\log \left(S_{x} / S_{r}\right)=[-6,-6.5]$. Pertaining to the unbalanced detection rates at $37 \mathrm{GHz}$, LBLs are overrepresented compared to HBLs in the plot. Only $14 \%$ of the objects are HBLs. When considered separately, LBLs, IBLs, and HBLs move 


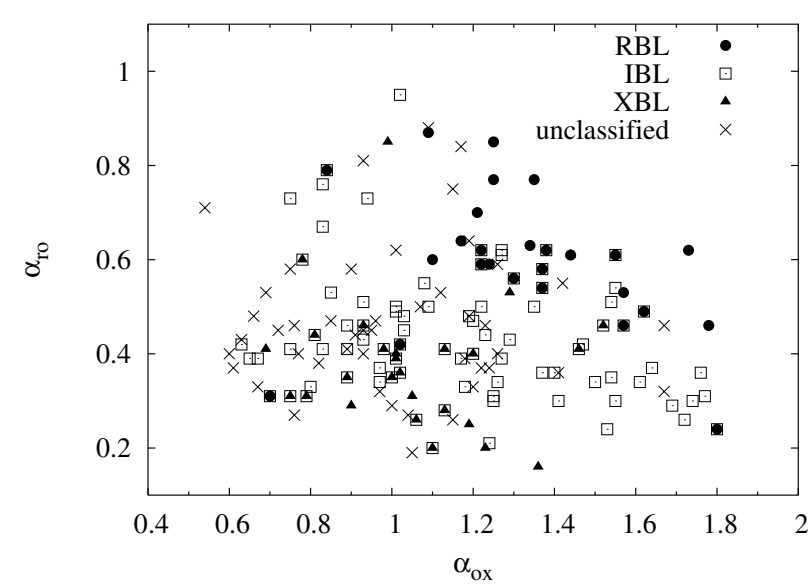

Fig. 8. The colour plane: $\alpha_{\text {ro }}$ plotted against $\alpha_{\text {ox }}$. Frequency intervals $5 \mathrm{GHz}-5500 \AA-1 \mathrm{keV}$, ROSAT band. Different symbols denote the observational classification of BL Lacs.

progressively from low to higher values of $\log \left(S_{x} / S_{r}\right)$. There is substantial overlap between the distributions. The average values are $-6.35,-5.50$, and -4.85 for LBLs, IBLs, and HBLs respectively.

\subsection{Broad band spectral indices}

\subsubsection{The $\alpha_{\text {ro }}-\alpha_{\text {ox }}$ diagram}

We calculated broad band spectral indices $\alpha_{\text {ro }}$ and $\alpha_{\text {ox }}$ to plot the sample in $\alpha_{\text {ro }}$ vs. $\alpha_{\text {ox }}$ diagram. Here the spectral index is defined as

$\alpha_{1-2}=-\frac{\log \left(S_{1} / S_{2}\right)}{\log \left(v_{1} / v_{2}\right)}$

where $S_{1}$ and $S_{2}$ are the fluxes in frequencies $v_{1}$ and $v_{2}$, respectively, and $S_{v}=v^{-\alpha}$.

The $\alpha_{\text {ro }}$ vs. $\alpha_{\text {ox }}$ diagram has also been used as a means to demonstrate the division of the population. Generally, XBLs have lower values for both indices occupying the lower left corner of the plot, whereas RBLs lie in the upper right corner (Stocke et al. 1985; Laurent-Muehleisen et al. 1999). IBLs seem to have bridged the gap with intermediate values of both indices.

For comparison, we plotted two diagrams; one with low radio frequency $(5 \mathrm{GHz})$ (Fig. 8) and one with high radio frequency (37 GHz) (Fig. 9). The optical wavelength used was $5500 \AA$. In both diagrams we used X-ray data at $1 \mathrm{keV}$ from the literature and, in addition, data from ROSAT band $0.1-2.4 \mathrm{keV}$. The error produced by the bandwidth difference is small compared with the benefits of a larger number of datapoints.

When the lower radio frequency is used (Fig. 8), the indices for the whole population are $\alpha_{\mathrm{ox}}=0.54-1.8$ and $\alpha_{\text {ro }}=$ 0.16-0.95. RBLs and XBLs occupy their expected locations, but overall the distribution is even.

As for the higher radio frequency diagram (Fig. 9), the intervals are slightly different: $\alpha_{\text {ox }}=0.63-1.8$ and $\alpha_{\text {ro }}=$ $0.29-1.13$. The values of $\alpha_{\text {ox }}$ are the same as in Fig. 8, but the number of datapoints is smaller. Here we note again the lack of

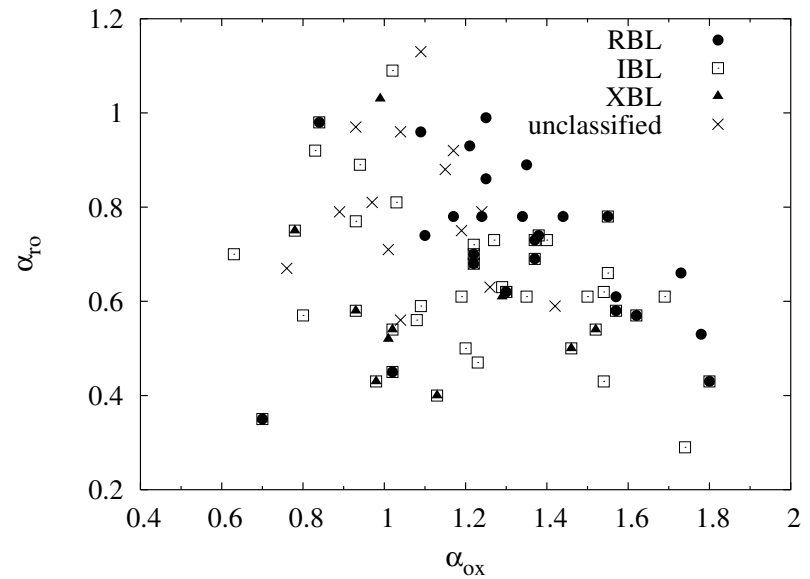

Fig. 9. The colour plane: $\alpha_{\text {ro }}$ plotted against $\alpha_{\mathrm{ox}}$. Frequency intervals $37 \mathrm{GHz}-5500 \AA-1 \mathrm{keV}$, ROSAT band. Different symbols denote the observational classification of BL Lacs.

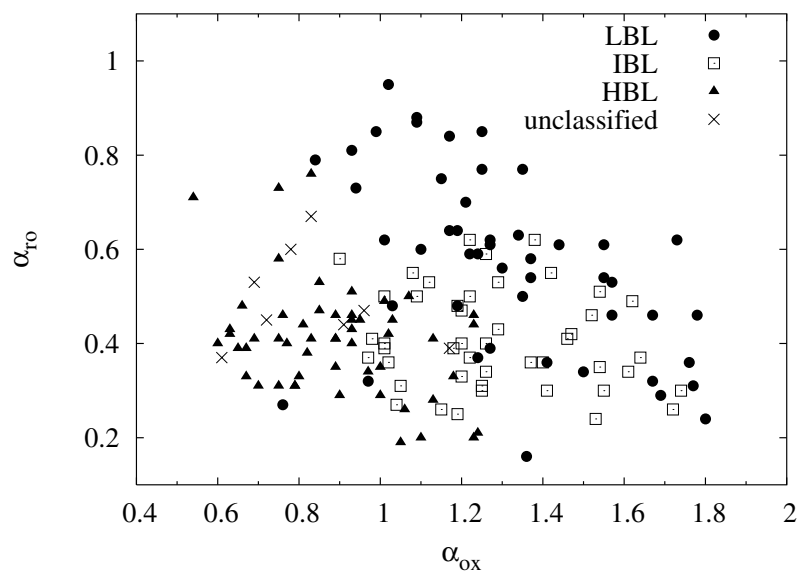

Fig. 10. The colour plane: $\alpha_{\text {ro }}$ plotted against $\alpha_{\text {ox }}$. Frequency intervals $5 \mathrm{GHz}-5500 \AA-1 \mathrm{keV}$, ROSAT band. Different symbols denote the physical classification of BL Lacs obtained in this study.

HBLs compared to LBLs. This is why the lower left corner of Fig. 9 is underpopulated compared with Fig. 8.

We also plotted the diagram with $5 \mathrm{GHz}$ showing the LBL/IBL/HBL classification of our study (Fig. 10). It clearly shows how the transition of the synchrotron peak from LBL to HBL moves the object on the $\alpha_{\text {ro }}-\alpha_{\text {ox }}-$ plane from the top to lower right and onwards to lower left, as described by Padovani \& Giommi (1995). However, there are several LBLs that appear on the wrong side of the $\alpha_{\mathrm{rx}}=0.75$ line that has usually been thought of as a dividing line between LBLs and HBLs. This suggests that the $\alpha_{\mathrm{rx}}=0.75$ divide is not very effective and LBLs take on very scattered values of $\alpha_{\text {ro }}$.

There are some objects in the $\alpha_{\text {ro }}-\alpha_{\text {ox }}$-plot (Fig. 10) with no classification (marked with $\mathrm{x}$ ). They are concentrated in quite a small area with $\alpha_{\mathrm{ox}}=0.61-1.17$ and $\alpha_{\text {ro }}=0.37-0.67$. Their synchrotron peak frequencies have not been calculated on account of very poor fits, but judging by their spectral indices they are likely to be HBLs. 


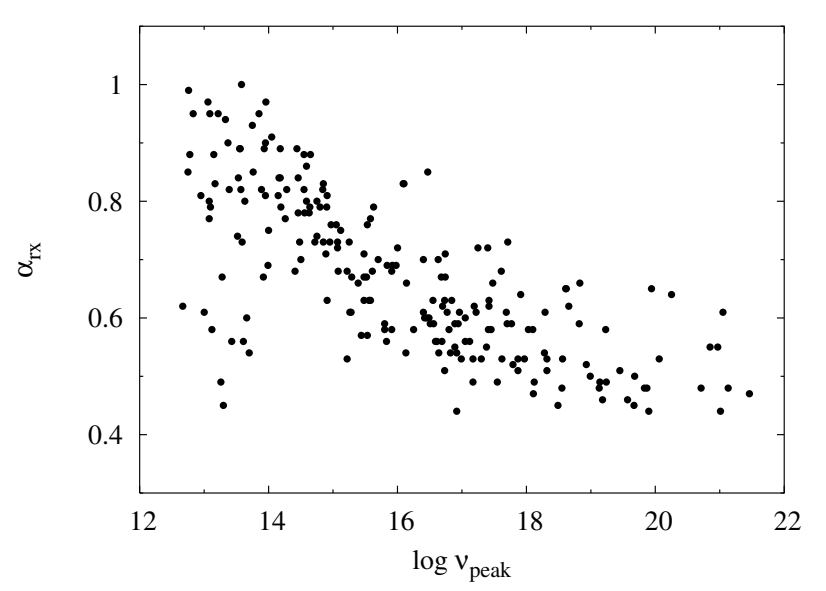

Fig. 11. Radio-X-ray spectral index $\alpha_{\text {rx }}$ plotted against synchrotron peak frequency.

\subsection{2. $\alpha_{\mathrm{rx}}$ Vs. $\log v_{\text {peak }}$}

The relation between $\alpha_{\mathrm{rx}}$ and $\log \nu_{\text {peak }}$ is shown in Fig. 11. We used radio frequency $5 \mathrm{GHz}$, and X-ray data comes primarily from $1 \mathrm{keV}$. For those objects that have no $1 \mathrm{keV}$ flux available, we used data from the ROSAT band. According to the Spearman rank correlation test, the negative correlation is significant at a $99 \%$ level. However, the correlation seems to break apart at $\log v_{\text {peak }}<14$. LBLs take on $\alpha_{\mathrm{rx}}$-values between 0.45-1. Fossati et al. (1998) suggested that the correlation between the radio luminosity and X-ray luminosity in the rising Compton-component makes $\alpha_{\text {rx }}$ tend to a fixed value when $\log v_{\text {peak }}<14$, but in our plot this seems not to be the case.

When $\log v_{\text {peak }}>14$, the correlation is apparent. This speaks for the authenticity of the UHBL candidates, as they take their place at the end of the continuum with low spectral index values. All objects with $\log v_{\text {peak }}>19$ also have $\alpha_{\mathrm{rx}} \leq 0.65$.

From the $\alpha_{\mathrm{rx}}$ vs. $\log v_{\text {peak }}$-plot, we can see that assigning an object an LBL/HBL classification based only on the value of $\alpha_{\mathrm{rx}}$ is risky. While an object with $\alpha_{\mathrm{rx}}>0.75$ is very likely an LBL, the opposite does not hold. In fact, approximately $30 \%$ of objects with $\alpha_{\text {rx }}<0.75$ have $\log v_{\text {peak }}<16$. At $\alpha_{\text {rx }} \approx 0.6$ the possible values of $v_{\text {peak }}$ span as much as eight magnitudes.

When compared with the corresponding figures of Fossati et al. (1998) and Padovani et al. (2003), our plot seems to be a combination of the two. This is explained by the fact that both the samples used by Fossati et al. (1 Jy and Slew Survey) and part of the sample used by Padovani et al. (DXRBS) are included in the Metsähovi sample. Thus we see a strong correlation with a substantial increase in scatter of the $\alpha_{\mathrm{rx}}$-values towards the lower end. We stress that our data considerably extends the range of values of $\log v_{\text {peak }}$.

\subsection{3. $\alpha_{\text {ro }}$ vs. $\log v_{\text {peak }}$}

The correlation between (i) the spectral index between radio (5 GHz) and optical (5500 ̊) frequencies and (ii) synchrotron peak frequency is shown in Fig. 12. The overall trend is

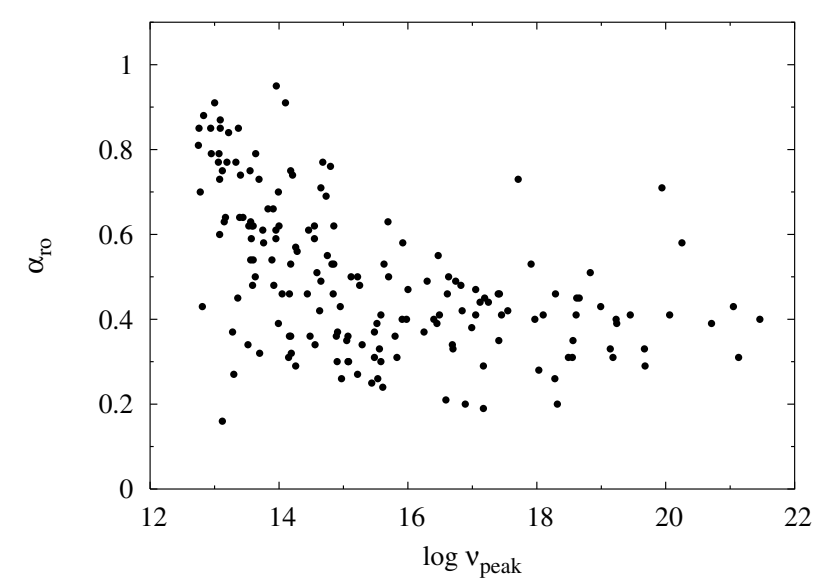

Fig. 12. Radio-optical spectral index $\alpha_{\text {ro }}$ plotted against synchrotron peak frequency.

easily seen as it changes from the steep negative correlation of LBLs to an almost constant trend of HBLs. The change occurs roughly when the synchrotron peak of the SED moves to frequencies higher than those used to calculate $\alpha_{\text {ro }}$. After that point, the peak frequency no longer has an influence on $\alpha_{\text {ro }}$. The negative correlation is significant at a 99\% level. Again, however, we see a few points breaking this scenario, both LBLs with very low values of $\alpha_{\text {ro }}$ and HBLs with high values of $\alpha_{\text {ro }}$.

We note that, as in the case of $\alpha_{\mathrm{rx}}$, the scatter in the figure is significant. Therefore the value of $\alpha_{\text {ro }}$ cannot be reliably used to classify BL Lacs. Only if $\alpha_{\text {ro }}$ is very close to one, the object is likely to be an LBL.

\section{Discussion}

Our findings support the results of Costamante et al. (2001). Their study concludes that objects $1 \mathrm{ES} 0033+595$, 1ES $0120+340$, 1ES $1218+304$, and 1ES 1426+428 are extreme HBLs with synchrotron peak frequencies exceeding $10^{18} \mathrm{~Hz}$. Our results show that they all peak at roughly $\log v_{\text {peak }} \approx 19$. Meanwhile, the extreme nature of 1ES 2344+514 (Giommi et al. 2000) is not revealed in our study, as it had a peak frequency of $\log v_{\text {peak }}=16.4$. Giommi et al. state that it is indeed very variable, with synchrotron peak frequencies ranging from $\log v_{\text {peak }} \approx 17$ to $\log v_{\text {peak }} \approx 18$. Mrk 501 is another example of a variable source; its synchrotron peak has been as high as $\log v_{\text {peak }} \approx 19$ (Pian et al. 1998 ), but the peak in our SED occured at $\log v_{\text {peak }} \approx 16.84$.

In this paper we tested the correlation between the synchrotron peak frequency and luminosities at radio, optical, and $\mathrm{X}$-ray wavelengths, along with luminosity at the synchrotron peak of each source. According to the blazar sequence scenario promoted by Fossati et al. (1998), HBLs have lower luminosities at all wavelenghts. In the X-ray region, in the SED of LBLs the synchrotron and SSC-components meet, resulting in a concave X-ray spectrum. In HBLs the synchrotron component peaks at or near the X-ray energies. Because the SEDs of LBLs and HBLs are so differently shaped in this frequency band, the $\mathrm{X}$-ray correlation may be less significant. Still, on the whole, LBLs are expected to be more luminous. When the defined 
frequency bands are considered, our data seems to support this scenario in all other frequencies except in X-rays. There we find a clear positive correlation that contradicts the blazar sequence. Luminosities rise towards the high peak frequencies. When all luminosity vs. $\log v_{\text {peak }}$ correlations are studied simultaneously, a kind of sequence is revealed: at radio frequencies the negative correlation is steep, in optical wavelengths the correlation is only slightly negative, and in the X-ray region it turns positive. However, the negative correlation of luminosity at $5 \mathrm{GHz}$ and synchrotron peak frequency is expected to originate solely from the shifting of the bulk of the synchrotron emission to the higher frequencies with growing $\log v_{\text {peak }}$ and, as such, does not authenticate the interdependence of source luminosity and peak frequency. A negative luminosity correlation at $5 \mathrm{GHz}$ and a positive one at X-ray energies is what we would expect, if the only parameter to change in the SED sequence was $v_{\text {peak }}$. When source luminosities at synchrotron peak frequencies are calculated, we find that they do not correlate with $\log v_{\text {peak }}$.

In addition to the positive correlation of X-ray luminosity and synchrotron peak and the lack of synchrotron luminosity correlation, the blazar sequence scenario is brought into question by the appearance of numerous low-luminosity LBLs at $5 \mathrm{GHz}$. The faintest LBLs reach even lower luminosities than most of the HBLs. As the $5 \mathrm{GHz}$ flux limit of even the least limited radio-selected sample, RGB, is $20 \mathrm{mJy}$, it is obvious that these objects have been identified for the most part from X-ray surveys. We note that there are some $5 \mathrm{GHz}$ low-luminosity LBLs that have a detection at $37 \mathrm{GHz}$. Especially for these sources, we have to consider the possibility that the $5 \mathrm{GHz}$ flux measurement is from a particularly quiescent state and does not represent the object accurately. Padovani et al. (2003) found low-luminosity LBLs among the DXRBS BL Lacs. In our lowluminosity LBLs there are 4 objects from the DXRBS survey.

The $5 \mathrm{GHz}$ low-luminosity LBLs also appear in luminosity plots from other frequency bands, again at the low-luminosity end. All the extreme HBLs have relatively high or intermediate luminosities. This effect, especially in X-rays, seems to support the scenario that UHBLs are in fact a rare class. If they were common and X-ray luminous, the numerous X-ray surveys performed lately should have detected them in large numbers. On the whole, the scatter in luminosities diminishes towards higher peak frequencies at all wavelengths. There is no solid evidence of truly high-luminosity HBLs with a measured redshift in radio wavelengths. The high-luminosity HBLs reported by Giommi et al. (2005) are from the Sedentary Survey, which is not included in our sample.

We find that the low-luminosity LBLs also behave abnormally on the broad band spectral index plots, $\alpha_{\mathrm{ro}}$ vs. $\alpha_{\mathrm{ox}}$, $\alpha_{\text {rx }}$ vs. $\log v_{\text {peak }}$, and $\alpha_{\text {ro }}$ vs. $\log v_{\text {peak }}$. In the first the BL Lac classes take their expected places except for a few of the lowluminosity LBLs that invade the region usually populated by HBLs. Doing so, they cross the $\alpha_{\mathrm{rx}}=0.75$ border frequently used to classify BL Lacs to LBLs and HBLs.

When the $\alpha_{\text {rx }}$ vs. $\log v_{\text {peak }}$-plot is examined, we find a strong negative correlation, but LBLs $\left(\log v_{\text {peak }}<14\right)$ disrupt the trend and take on values $\alpha_{\mathrm{rx}}=0.45-1$. Hints of such a distribution was discovered by Padovani et al. (2003), but the wider range of $\log v_{\text {peak }}$ in our sample makes it evident. All LBLs with conspicuously low $\alpha_{\mathrm{rx}}$ are among the low-luminosity LBLs in the $5 \mathrm{GHz}$ luminosity plot. We considered the possibility that the low indices in these objects are due to the wider bandwidth of the ROSAT fluxes compared to the $1 \mathrm{keV}$ fluxes, but this seems to have little effect. For those objects that had $\alpha_{\text {rx }}$ computed for both X-ray energies, we calculated the average values of both indices. The average for ROSAT band $\alpha_{\text {rx }}$ was 0.63 , while for $1 \mathrm{keV}$ fluxes it was 0.69 . This difference is small compared to the deviation of the low- $\alpha_{\text {rx }}$ LBLs from their expected location. Also for 3 of the 10 LBLs with lowest $\alpha_{\mathrm{rx}}$-values, $1 \mathrm{keV}$ flux has been used in calculating the spectral index.

In the $\alpha_{\text {ro }}$ vs. $\log v_{\text {peak }}-$ plot, LBLs behave in a similar way; at $\log v_{\text {peak }} \approx 13$ they have $\alpha_{\text {ro }}=0.16-0.95$. In this plot we also see 3 HBLs with conspicuously high $\alpha_{\text {ro }}$. They have relatively high radio luminosities and low optical luminosities. Each source has only one flux measurement at $5500 \AA$, possibly from an anomalously quiescent state. For these HBLs we also considered the possibility that their X-ray flux actually originates from IC rather than synchrotron radiation. This would lower their peak frequencies and move them left on the $\alpha_{\text {ro }}$ vs. $\log v_{\text {peak }}$-plane. For two of them (RXS J1456.0+5048, $\log v_{\text {peak }}=19.94$ and RXS J1410.5+6100, $\left.\log v_{\text {peak }}=20.25\right)$, this seems possible albeit uncertain.

As explained before, when there are few datapoints representing the SED, the peak frequency is easily overestimated in the case of extreme HBLs. This also affects the luminosity correlations. However we do not expect the significance of the correlations at defined wavelengths to change, even if the datapoints in the high-energy end moved to the left. The only case where the consequences could be substantial is the $\log L_{\text {peak }}$ vs. $\log v_{\text {peak }}$-plot (Fig. 6). If the peak frequency is exaggerated, so is the luminosity at peak frequency. Thus the uncertain datapoints can move from upper right to lower left, possibly changing the correlation. We tested this by artificially lowering the peak luminosities of those sources whose $v F_{\text {peak }}$ was notably higher in the SED than their X-ray flux, $v F_{\mathrm{X} \text {-ray }}$. We changed their peak frequencies accordingly, and the highest $\log v_{\text {peak }}$ in the sample changed from 21.46 to 19.44 , yet the overall shape of the plot did not change enough to produce significant correlation. Therefore we find it unlikely that this overestimation of the $\log v_{\text {peak }}$ of HBLs affects the main results of this study.

\section{Conclusions}

In this paper we collected a large amount of multifrequency data, as well as new flux measurements at $37 \mathrm{GHz}$, and plotted the spectral energy distributions of over 300 BL Lacs of the Metsähovi BL Lac sample. Using such an extensive sample allowed us to detect the whole range of synchrotron peak frequencies reaching up to the $\mathrm{MeV}$-region. The main conclusions are as follows:

1. For 22 objects we find that $\log v_{\text {peak }}>19$. This highfrequency tail of the $v_{\text {peak }}$ distribution is unrepresented in 
most previous studies, but we find it essential for determining the properties of the population accurately.

2. The positive correlation of X-ray luminosity and synchrotron peak frequency and the lack of correlation between the source luminosity at synchrotron peak and peak frequency contradict the blazar sequence scenario. The differences between LBLs and HBLs cannot be attributed to unequal luminosities.

3. When the broad band spectral indices $\alpha_{\text {rx }}$ and $\alpha_{\text {ro }}$ are plotted against $\log v_{\text {peak }}$, we find substantial scatter in the figures. This implies that the values of $\alpha_{\text {rx }}$ and $\alpha_{\text {ro }}$ cannot be reliably used in BL Lac classification.

4. Based on the smooth, declining distribution of $v_{\text {peak }}$ and the lack of any bimodality in all other tests and calculations, we conclude that the BL Lac population as a whole is continuous and undivided.

Acknowledgements. We gratefully acknowledge the funding from the Academy of Finland for our Metsähovi and SEST observing projects (project numbers 205969, 46341, and 51436). E.N. thanks Ilona Torniainen for help in producing the SED plots for publication.

\section{References}

Bondi, M., Marchã, M. J. M., Dallacasa, D., \& Stanghellini, C. 2001, MNRAS, 325, 1109

Caccianiga, A., Maccacaro, T., Wolter, A., della Ceca, R., \& Gioia, I. M. 1999, ApJ, 513, 51

Caccianiga, A., \& Marchã, M. J. M. 2004, MNRAS, 348, 937

Costamante, L., Ghisellini, G., Giommi, P., et al. 2001, A\&A, 371, 512

Donato, D., Ghisellini, G., Tagliaferri, G., \& Fossati, G. 2001, A\&A, 375, 739

Fossati, G., Maraschi, L., Celotti, A., Comastri, A., \& Ghisellini, G. 1998, MNRAS, 299, 433
Ghisellini, G. 1999, ApL\&C, 39, 17

Gioia, I., Maccacaro, T., Schild, R., et al. 1990, ApJS, 72, 567

Giommi, P., Ansari, S. G., \& Micol, A. 1995, A\&AS, 109, 267

Giommi, P., Padovani, P., \& Perlman, E. 2000, MNRAS, 317, 743

Giommi, P., Ghisellini, G., Padovani, P., \& Tagliaferri, G. 2001, AIPC, 599, 441

Giommi, P., Piranomonte, S., Perri, M., \& Padovani, P. 2005, A\&A, 434,385

Hartman, R. C., Bertsch, D. L., Bloom, S. D., et al. 1999, ApJS, 123, 79

Jannuzi, B. T., Smith, P. S., \& Elston, R. 1994, ApJ, 428, 130

Kühr, H., \& Schmidt, G. 1990, AJ, 99, 1

Lamer, G., Brunner, H., \& Staubert, R. 1996, A\&A, 311, 384

Landt, H., Padovani, P., Perlman, E. S., et al. 2001, MNRAS, 323, 757

Laurent-Muehleisen, S. A., Kollgaard, R. I., Feigelson, E. D., Brinkmann, W., \& Siebert, J. 1999, ApJ, 525, 127

Padovani, P., \& Giommi, P. 1995, ApJ, 444, 567

Padovani, P., Perlman, E. S., Landt, H., Giommi, P., \& Perri, M. 2003, ApJ, 588, 128

Perlman, E. S., Padovani, P., Giommi, P., et al. 1998, AJ, 115, 1253

Perlman, E. S., \& Stocke, J. T. 1993, ApJ, 406, 430

Perlman, E. S., Stocke, J. T., Schachter, J. F., et al. 1996, ApJS, 104, 251

Pian, E., Vacanti, G., Tagliaferri, G., et al. 1998, ApJ, 492, 17

Stickel, M., \& Kühr, H. 1994, A\&AS, 103, 349

Stickel, M., Padovani, P., Urry, C. M., Fried, J. W., \& Kühr, H. 1991, ApJ, 374, 431

Stocke, J. T., Liebert, J., Schmidt, G., et al. 1985, ApJ, 298, 619

Stocke, J. T., Morris, S. L., Gioia, I. M., et al. 1991, ApJS, 76, 813

Urry, C. M., \& Padovani, P. 1995, PASP, 107, 715

Veron-Cetty, M. P., \& Veron, P. 2000, ESOSR, 19, 1

Veron-Cetty, M. P., \& Veron, P. 2001, A\&A, 374, 92

Veron-Cetty, M. P., \& Veron, P. 2003, A\&A, 412, 399

Voges, W., Aschenbach, B., Boller, T., et al. 1999, A\&A, 349, 389

White, N. E., Giommi, P., \& Angelini, L. 1996, VizieR Online Data Catalogue IX/12, 9012, 0 


\section{Online Material}


Table 3. The Metsähovi BL Lac sample. The synchrotron peak frequency and subsequent classification designated in this study are included when available.

\begin{tabular}{|c|c|c|c|c|}
\hline Source & $\begin{array}{l}\mathrm{RA}(\mathrm{J} 2000) \\
\end{array}$ & $\operatorname{Dec}(\mathbf{J} 2000)$ & $\log v_{\text {peak }}$ & Class \\
\hline NRAO 5 & $00: 06: 13.9$ & $-06: 23: 36$ & 12.75 & LBL \\
\hline RX J0007.9+4711 & 00:07:59.9 & 47:12:07 & 16.14 & IBL \\
\hline MS 0011.7+0837 & $00: 14: 19.7$ & 08:54:04 & 16.74 & HBL \\
\hline RXS J0018.4+2947 & $00: 18: 27.8$ & $29: 47: 32$ & - & - \\
\hline PKS 0017+200 & $00: 19: 37.9$ & $20: 21: 46$ & 13.08 & LBL \\
\hline PKS 0019+058 & $00: 22: 32.5$ & 06:08:05 & 13.19 & LBL \\
\hline RXS J0325.2+1515 & $00: 35: 14.9$ & $15: 15: 04$ & 13.73 & LBL \\
\hline 1ES $0033+595$ & $00: 35: 52.6$ & 59:50:04 & 18.93 & HBL \\
\hline 1ES $0037+405$ & $00: 40: 13.8$ & 40:50:04 & 16.8 & HBL \\
\hline RXS J0045.3+2127 & $00: 45: 19.1$ & $21: 27: 43$ & 16.89 & HBL \\
\hline B3 0045+395 & $00: 47: 55.2$ & 39:48:57 & - & - \\
\hline EXO 0044.4+2001 & 00:47:08 & $20: 17: 44$ & 16.05 & IBL \\
\hline PKS 0047+023 & $00: 49: 43.3$ & 02:37:04 & 13.56 & LBL \\
\hline PKS 0048-097 & $00: 50: 41.2$ & $-09: 29: 06$ & 13.39 & LBL \\
\hline NPM1G -09.0033 & 00:56:20 & $-09: 36: 32$ & - & - \\
\hline RXS J0058.2+1723 & $00: 58: 16.8$ & $17: 23: 14$ & - & - \\
\hline Q J0109+181 & 01:09:08.1 & $18: 16: 03$ & - & _- \\
\hline NPM1G +41.0022 & 01:10:04.8 & 41:49:50 & 17.7 & HBL \\
\hline RXS J0111.5+0536 & 01:11:30.1 & $05: 36: 28$ & - & - \\
\hline S2 $0109+22$ & 01:12:05.8 & $22: 44: 39$ & 13.59 & LBL \\
\hline RXS J0115.7+2519 & $01: 15: 46.5$ & 25:19:57 & 13.43 & LBL \\
\hline 1ES $0120+340$ & 01:23:08.7 & $34: 20: 51$ & 18.32 & HBL \\
\hline MS 0122.1+0903 & $01: 24: 44.5$ & 09:18:49 & 15.53 & IBL \\
\hline B3 $0133+388$ & 01:36:32.6 & 39:06:00 & 16.59 & HBL \\
\hline PKS 0139-09 & $01: 41: 25.8$ & $-09: 28: 43$ & 13.4 & LBL \\
\hline 1ES $0145+138$ & 01:48:29.7 & 14:02:18 & 15.44 & IBL \\
\hline NPM1G +01.0067 & 01:52:39.6 & 01:47:17 & - & - \\
\hline $8 \mathrm{C} 0149+710$ & $01: 53: 25.8$ & $71: 15: 07$ & 14.75 & IBL \\
\hline RXS J0155.9+1502 & 01:56:00.3 & $15: 02: 13$ & - & - \\
\hline $87 \mathrm{~GB} 01569+1032$ & 01:59:34.4 & 10:47:07 & 15.56 & IBL \\
\hline RXS J0200.4+2712 & 02:00:29.5 & $27: 12: 36$ & - & - \\
\hline MS 0158.5+0019 & 02:01:06.1 & 00:34:00 & 17.87 & HBL \\
\hline RXS J0202.4+0849 & 02:02:26.4 & $08: 49: 12$ & - & - \\
\hline S5 0159+72 & 02:03:33.3 & $72: 32: 53$ & 13.99 & LBL \\
\hline MS 0205.7+3509 & 02:08:38.2 & $35: 23: 13$ & 15.22 & IBL \\
\hline 87 GB $02109+5130$ & $02: 14: 17.9$ & $51: 44: 52$ & 17.69 & HBL \\
\hline RXS J0216.5+2314 & $02: 16: 32.1$ & $23: 14: 47$ & - & - \\
\hline Z 0214+083 & 02:17:17 & 08:37:03 & 15.23 & IBL \\
\hline S4 $0218+35$ & 02:21:05.4 & $35: 56: 15$ & - & - \\
\hline $3 \mathrm{C} 66 \mathrm{~A}$ & $02: 22: 39.6$ & 43:02:08 & 15.63 & IBL \\
\hline RXS J0227.2+0201 & $02: 27: 16.6$ & 02:01:58 & - & - \\
\hline 1ES $0229+200$ & $02: 32: 48.6$ & $20: 17: 17$ & 19.45 & HBL \\
\hline Q $0230+3429$ & $02: 33: 20.3$ & $34: 42: 54$ & 15.84 & IBL \\
\hline AO $0235+164$ & $02: 38: 38.8$ & $16: 36: 59$ & 13.57 & LBL \\
\hline S5 0238+71 & $02: 43: 31$ & $71: 20: 18$ & 16.3 & IBL \\
\hline NPM1G +10.0097 & $02: 45: 13.5$ & $10: 47: 23$ & - & - \\
\hline RXS J0250.6+1712 & 02:50:38 & 17:12:08 & - & - \\
\hline MS 0257.9+3429 & 03:01:03.8 & $34: 41: 01$ & 13.28 & LBL \\
\hline 4C 47.08 & $03: 03: 35.2$ & $47: 16: 17$ & 14.18 & LBL \\
\hline RXS J0303.5+0554 & 03:03:30.1 & $05: 54: 17$ & - & - \\
\hline PKS 0306+102 & 03:09:03.6 & $10: 29: 16$ & 12.94 & LBL \\
\hline VZw331 & 03:13:57.9 & $41: 15: 24$ & 14.48 & LBL \\
\hline RXS J0314.0+2445 & 03:14:02.7 & $24: 44: 31$ & 12.67 & LBL \\
\hline
\end{tabular}


Table 3. continued.

\begin{tabular}{|c|c|c|c|c|}
\hline Source & $\mathrm{RA}(\mathrm{J} 2000)$ & $\operatorname{Dec}(\mathrm{J} 2000)$ & $\log v_{\text {peak }}$ & Class \\
\hline RXS J0314.3+0620 & $03: 14: 23.9$ & $06: 19: 57$ & 19.57 & HBL \\
\hline RXS J0316.1+0904 & 03:16:12.9 & 09:04:43 & 15.91 & IBL \\
\hline MS 03170+1834 & $03: 19: 51.8$ & $18: 45: 35$ & 16.99 & HBL \\
\hline RGB J0321+2336 & 03:22:00 & $23: 36: 11$ & - & - \\
\hline $2 \mathrm{E} 0323+0214$ & $03: 26: 13.9$ & $02: 25: 14$ & 19.87 & HBL \\
\hline RXS J0331.3+0654 & $03: 31: 19.4$ & $06: 54: 28$ & - & - \\
\hline RXS J0349.9+0640 & $03: 49: 59.7$ & $06: 40: 56$ & - & - \\
\hline PKS 0406+121 & 04:09:22.1 & $12: 17: 39$ & 13.22 & LBL \\
\hline 2E $0414+0057$ & $04: 16: 52.4$ & $01: 05: 24$ & 20.71 & HBL \\
\hline 1WGA J0421.5+1433 & $04: 21: 33.1$ & $14: 33: 54$ & 13.93 & LBL \\
\hline MS 0419.3+1943 & $04: 22: 18.5$ & $19: 50: 53$ & 16.82 & HBL \\
\hline PKS 0420+022 & $04: 22: 52.2$ & 02:19:27 & - & - \\
\hline PKS 0422+004 & $04: 24: 46.8$ & 00:36:07 & 15.69 & IBL \\
\hline MCG 38364 & $04: 25: 51.3$ & $-08: 33: 38$ & 17.17 & HBL \\
\hline 2EG J0432+2910 & $04: 33: 37.7$ & 29:05:56 & 14.09 & LBL \\
\hline 1ES 0446+449 & 04:50:07.3 & $45: 03: 12$ & - & - \\
\hline PKS 0459+135 & $05: 02: 33.2$ & 13:38:11 & 13.55 & LBL \\
\hline Q $0458+6530$ & 05:03:03.4 & $65: 34: 10$ & 18.12 & HBL \\
\hline RXS J0505.5+0416 & 05:05:34.7 & $04: 15: 54$ & 16.94 & HBL \\
\hline 1ES $0502+675$ & 05:07:56.1 & $67: 37: 24$ & 19.18 & HBL \\
\hline S5 0454+84 & 05:08:42.5 & $84: 32: 05$ & 13.58 & LBL \\
\hline MG 0509+0541 & 05:09:25.9 & $05: 41: 35$ & 15.34 & IBL \\
\hline 4U 0506-03 & 05:09:39 & $-04: 00: 36$ & 17.94 & HBL \\
\hline $2 \mathrm{E} 0514+0626$ & 05:17:04 & $06: 29: 39$ & - & - \\
\hline 1ES $0525+713$ & 05:31:41.7 & $71: 22: 17$ & - & - \\
\hline TEX 0554+534 & 05:58:11.6 & $53: 28: 19$ & 14.44 & LBL \\
\hline MS 0607.9+7108 & $06: 13: 42.8$ & 71:07:29 & 14.85 & IBL \\
\hline 87GB 06216+4441 & $06: 25: 18.3$ & 44:40:02 & 13.61 & LBL \\
\hline 1ES $0647+250$ & $06: 50: 46.5$ & 25:03:00 & 18.28 & $\mathrm{HBL}$ \\
\hline B3 $0651+428$ & $06: 54: 43.5$ & $42: 47: 59$ & 15.12 & IBL \\
\hline NPM1G +42.0131 & 06:56:10.6 & 42:37:02 & 17.25 & $\mathrm{HBL}$ \\
\hline EXO 0706.1+5913 & 07:10:30.1 & 59:08:21 & 21.05 & HBL \\
\hline RXS J0712.3+5719 & $07: 12: 18.7$ & $57: 19: 22$ & - & - \\
\hline S5 $0716+714$ & $07: 21: 53.3$ & 71:20:36 & 14.46 & LBL \\
\hline RXS J0723.2+5841 & $07: 23: 13.2$ & $58: 41: 23$ & - & - \\
\hline FIRST J0724.7+2621 & $07: 24: 42.8$ & $26: 21: 30$ & 16.39 & IBL \\
\hline PKS 0723-008 & $07: 25: 50.7$ & $-00: 54: 56$ & - & - \\
\hline FIRST J0730.4+3307 & 07:30:26.1 & $33: 07: 22$ & 16.29 & IBL \\
\hline RXS J0737.3+3517 & $07: 37: 21$ & $35: 17: 41$ & 17.77 & HBL \\
\hline FIRST J0738.6+3139 & $07: 38: 37.8$ & $31: 39: 30$ & - & - \\
\hline PKS 0735+17 & 07:38:07.4 & $17: 42: 19$ & 13.95 & LBL \\
\hline FIRST J0741.3+2253 & $07: 41: 18.8$ & $22: 53: 39$ & - & - \\
\hline MS 0737.9+7441 & 07:44:05.1 & $74: 33: 59$ & 13.61 & LBL \\
\hline S4 0749+54 & 07:53:01.3 & 53:53:00 & 13.12 & LBL \\
\hline GB $0751+485$ & $07: 54: 45.7$ & $48: 23: 51$ & 14.32 & LBL \\
\hline PKS 0754+100 & 07:57:06.7 & $09: 56: 35$ & 13.63 & LBL \\
\hline RXS J0800.1+6210 & 08:00:06.5 & $62: 10: 12$ & - & - \\
\hline RXS J0801.0+6444 & 08:01:00.7 & $64: 44: 43$ & - & - \\
\hline RXS J0805.4+7534 & $08: 05: 26.5$ & $75: 34: 25$ & 15.96 & IBL \\
\hline SBS 0802+596 & 08:06:25.9 & $59: 31: 07$ & 16.69 & HBL \\
\hline B2 0806+31 & 08:09:13.4 & $31: 22: 22$ & - & - \\
\hline RXS J0809.6+3455 & 08:09:38.5 & $34: 55: 37$ & 18.29 & HBL \\
\hline 1ES 0806+524 & 08:09:49.2 & $52: 18: 58$ & 16.56 & $\mathrm{HBL}$ \\
\hline PKS 0808+019 & $08: 11: 26.6$ & $01: 46: 52$ & 13.17 & LBL \\
\hline RXS J0812.1+5717 & 08:12:08.8 & $57: 17: 34$ & - & - \\
\hline
\end{tabular}


Table 3. continued.

\begin{tabular}{|c|c|c|c|c|}
\hline Source & $\mathrm{RA}(\mathrm{J} 2000)$ & $\operatorname{Dec}(\mathrm{J} 2000)$ & $\log v_{\text {peak }}$ & Class \\
\hline EXO 0811.2+2949 & $08: 14: 21.8$ & $29: 40: 32$ & - & - \\
\hline 1WGA J0816.0-0736 & 08:16:04.3 & $-07: 35: 57$ & 14.19 & LBL \\
\hline RXS J0816.3+5739 & $08: 16: 23.8$ & $57: 39: 03$ & 17.19 & HBL \\
\hline RXS J0816.6+6208 & 08:16:40.9 & $62: 08: 44$ & 15.25 & IBL \\
\hline OJ 425 & $08: 18: 16.1$ & $42: 22: 46$ & 13.33 & LBL \\
\hline FIRST J0818.4+2814 & $08: 18: 27.3$ & $28: 14: 02$ & 16.01 & IBL \\
\hline FIRST J0819.4+4037 & $08: 19: 25.8$ & $40: 37: 43$ & 16.61 & HBL \\
\hline FIRST J0820.3+3640 & 08:20:20.2 & $36: 40: 04$ & 14 & LBL \\
\hline $4 C 22.21$ & $08: 23: 24.8$ & $22: 23: 03$ & 13.09 & LBL \\
\hline PKS 0823+033 & $08: 25: 50.3$ & 03:09:24 & 13.08 & LBL \\
\hline RXS J0828.2+4153 & $08: 28: 14.2$ & $41: 53: 50$ & 18.66 & HBL \\
\hline B3 $0827+395$ & 08:30:19.4 & $39: 23: 47$ & - & - \\
\hline PKS 0829+046 & $08: 31: 48.9$ & 04:29:39 & 13.53 & LBL \\
\hline $1 \mathrm{H} 0827+089$ & $08: 31: 54.8$ & $08: 47: 58$ & 14.22 & LBL \\
\hline OJ 448 & $08: 32: 23.2$ & $49: 13: 21$ & 13.06 & LBL \\
\hline TEX 0836+182 & 08:39:30.7 & $18: 02: 47$ & 14.55 & IBL \\
\hline FIRST J0847.0+4117 & $08: 47: 02.5$ & $41: 17: 57$ & 18.11 & HBL \\
\hline RXS J0847.2+1133 & $08: 47: 12.9$ & $11: 33: 52$ & 19.13 & HBL \\
\hline RXS J0848.4+8111 & $08: 48: 27.8$ & $81: 11: 47$ & - & - \\
\hline US 1889 & 08:54:09.9 & 44:08:31 & 17.42 & HBL \\
\hline OJ 287 & $08: 54: 48.8$ & $20: 06: 30$ & 13.89 & LBL \\
\hline NPM1G -09.0307 & 09:08:02.2 & $-09: 59: 37$ & 15.52 & IBL \\
\hline B2 $0906+31$ & 09:09:53.3 & $31: 06: 03$ & 17.4 & HBL \\
\hline Ton 1015 & 09:10:37.1 & $33: 29: 24$ & 15.39 & IBL \\
\hline FIRST J0910.8+3902 & 09:10:52 & 39:02:02 & 18.33 & HBL \\
\hline B2 $0912+29$ & $09: 15: 52.4$ & $29: 33: 24$ & 16 & IBL \\
\hline RXS J0916.8+5238 & $09: 16: 52$ & $52: 38: 28$ & 17.22 & HBL \\
\hline MS 0922.9+7459 & 09:28:02.6 & $74: 47: 19$ & - & - \\
\hline RXS J0929.2+5013 & 09:29:15.4 & $50: 13: 35$ & 14.59 & IBL \\
\hline S5 0916+86 & 09:29:42.7 & $86: 12: 21$ & 14.16 & LBL \\
\hline 1ES $0927+500$ & 09:30:37.5 & $49: 50: 25$ & 21.13 & HBL \\
\hline B2 $0927+35$ & 09:30:55.3 & $35: 03: 38$ & 14.8 & IBL \\
\hline RXS J0930.9+3933 & 09:30:56.8 & $39: 33: 33$ & - & - \\
\hline SBS 0936+522 & 09:39:37.9 & $52: 01: 46$ & - & - \\
\hline B2 0937+26 & 09:40:13.6 & $26: 03: 26$ & 14.75 & IBL \\
\hline US 1015 & 09:50:11.8 & $45: 53: 20$ & 15.48 & IBL \\
\hline RGB J0952+656 & $09: 52: 32.2$ & $65: 38: 01$ & 15.08 & IBL \\
\hline MS 0950.9+4929 & 09:54:09.8 & $49: 15: 00$ & 16.92 & HBL \\
\hline S4 0954+65 & 09:58:47.2 & $65: 33: 54$ & 13.76 & LBL \\
\hline $4 C 22.25$ & $10: 00: 21.36$ & $22: 33: 07.4$ & - & - \\
\hline MS 0958.9+2102 & $10: 01: 42.4$ & $20: 48: 18$ & 15.54 & IBL \\
\hline EXO 1004.0+3509 & $10: 06: 56.3$ & $34: 54: 45$ & 16.92 & HBL \\
\hline RXS J10081+4705 & 10:08:11.3 & $47: 05: 20$ & 19.67 & HBL \\
\hline NRAO 350 & $10: 12: 13.3$ & $06: 30: 57$ & 16.09 & IBL \\
\hline RXS J1012.7+4229 & $10: 12: 44.3$ & $42: 29: 57$ & 20.97 & HBL \\
\hline GB $1011+496$ & $10: 15: 04.2$ & $49: 26: 01$ & 16.74 & HBL \\
\hline RXS J1016.2+4108 & $10: 16: 16.7$ & 41:08:13 & 16.62 & HBL \\
\hline RXS J1022.7-0112 & $10: 22: 43.9$ & $-01: 12: 56$ & 17.97 & HBL \\
\hline 1ES $1028+511$ & $10: 31: 18.5$ & $50: 53: 36$ & 18.56 & HBL \\
\hline FIRST J1032.6+3738 & $10: 32: 40.7$ & $37: 38: 26$ & - & - \\
\hline RXS J1037.7+5711 & $10: 37: 44.2$ & $57: 11: 57$ & 14.95 & IBL \\
\hline TEX 1040+244 & 10:43:09 & $24: 08: 35$ & 13.1 & LBL \\
\hline 1ES 1044+549 & $10: 47: 45.8$ & $54: 37: 41$ & 13 & LBL \\
\hline MS $1050.7+4946$ & $10: 53: 44.2$ & $49: 29: 54$ & 15.29 & IBL \\
\hline FIRST J1054.5+3855 & $10: 54: 31.8$ & $38: 55: 22$ & 16.68 & HBL \\
\hline
\end{tabular}


Table 3. continued.

\begin{tabular}{|c|c|c|c|c|}
\hline Source & $\overline{\mathrm{RA}(\mathrm{J} 2000)}$ & $\overline{\operatorname{Dec}(J 2000)}$ & $\log v_{\text {peak }}$ & Class \\
\hline RXS J1055.5-0126 & $10: 55: 34.1$ & $-01: 26: 05$ & - & - \\
\hline RXS J1056.1+0252 & $10: 56: 06.6$ & $02: 52: 13$ & - & - \\
\hline FIRST J1057.3+2303 & $10: 57: 23.1$ & $23: 03: 18$ & 18.7 & HBL \\
\hline RXS J1057.8+0059 & $10: 57: 52.4$ & $00: 59: 13$ & - & - \\
\hline FIRST J1058.4+2817 & $10: 58: 29.9$ & $28: 17: 46$ & 18.37 & HBL \\
\hline RXS J1058.6+5628 & $10: 58: 37.7$ & $56: 28: 12$ & 15.64 & IBL \\
\hline MC $1057+100$ & 11:00:20.2 & $09: 49: 35$ & - & - \\
\hline RXS J1100.3+4019 & 11:00:21.1 & $40: 19: 28$ & 18.76 & HBL \\
\hline RXS J1102.8-0148 & $11: 02: 52$ & $-01: 48: 51$ & - & - \\
\hline MRK 421 & $11: 04: 27.2$ & $38: 12: 32$ & 18.49 & HBL \\
\hline 1ES 1106+244 & 11:09:16.2 & $24: 11: 20$ & 16.91 & HBL \\
\hline OP $1106.7+3654$ & $11: 09: 33.5$ & $36: 38: 26$ & - & - \\
\hline FIRST J1110.9+3539 & $11: 10: 56.9$ & $35: 39: 06$ & - & - \\
\hline RXS J1110.6+7133 & $11: 10: 37.5$ & $71: 33: 57$ & 16.96 & HBL \\
\hline RXS J1111.5+3452 & 11:11:30.9 & $34: 52: 00$ & - & - \\
\hline FIRST J1117.6+2548 & $11: 17: 40.4$ & $25: 48: 46$ & 15.71 & IBL \\
\hline EXO $1118.0+4228$ & $11: 20: 48.1$ & $42: 12: 13$ & 17.41 & HBL \\
\hline US 2504 & $11: 29: 50.1$ & $26: 52: 53$ & 12.97 & LBL \\
\hline MS $1133.7+1618$ & $11: 36: 17.6$ & $16: 01: 53$ & 15.9 & IBL \\
\hline MRK 180 & $11: 36: 26.5$ & 70:09:28 & 18.61 & HBL \\
\hline RXS J1136.5+6737 & $11: 36: 30.1$ & $67: 37: 04$ & 17.55 & HBL \\
\hline FIRST J1136.8+2550 & $11: 36: 50.1$ & $25: 50: 52$ & 15.13 & IBL \\
\hline $2 \mathrm{E} 1146+2456$ & $11: 49: 29.9$ & $24: 38: 55$ & 17.87 & HBL \\
\hline EXO $1449.9+2455$ & $11: 49: 30.3$ & $24: 39: 27$ & 19.83 & HBL \\
\hline B2 $1147+245$ & 11:50:19.2 & $24: 17: 54$ & 13.95 & LBL \\
\hline RXS J1151.4+5859 & $11: 51: 24.6$ & $58: 59: 14$ & 16.4 & IBL \\
\hline FIRST J1152.1+2837 & $11: 52: 10.7$ & $28: 37: 21$ & 14.8 & IBL \\
\hline FIRST J1153.7+3823 & $11: 53: 42.9$ & $38: 23: 06$ & - & - \\
\hline MS $1154.1+4255$ & $11: 56: 46.6$ & $42: 38: 10$ & 14.91 & IBL \\
\hline B3 $1159+450$ & $12: 02: 08.6$ & $44: 44: 21$ & 15.92 & IBL \\
\hline B3 1206+416 & $12: 09: 22.8$ & 41:19:41 & 14.59 & IBL \\
\hline $1207+39 W 4$ & $12: 10: 26.6$ & 39:29:08 & - & - \\
\hline MS $1209.0+3917$ & $12: 11: 34.2$ & 39:00:55 & - & - \\
\hline 1ES 1212+078 & $12: 15: 10.9$ & $07: 32: 03$ & 15.91 & IBL \\
\hline Q $1214+1753$ & $12: 16: 56.9$ & $17: 37: 12$ & - & - \\
\hline B2 $1215+30$ & $12: 17: 52$ & 30:07:01 & 15.58 & IBL \\
\hline GB2 $1217+348$ & $12: 20: 08.4$ & $34: 31: 22$ & 14.46 & LBL \\
\hline PG 1218+304 & $12: 21: 21.9$ & $30: 10: 37$ & 19.14 & HBL \\
\hline ON 231 & $12: 21: 31.7$ & $28: 13: 58$ & 14.84 & IBL \\
\hline UM 493 & $12: 22: 06.5$ & $-01: 06: 38$ & - & - \\
\hline RXS J1222.2+3541 & $12: 22: 12.4$ & $35: 41: 00$ & 12.81 & LBL \\
\hline S5 $1221+80$ & $12: 23: 40.4$ & $80: 40: 04$ & 14.21 & LBL \\
\hline MS $1221.8+2452$ & $12: 24: 24.3$ & $24: 36: 24$ & 13.99 & LBL \\
\hline 1WGA J1225.3+1818 & $12: 25: 18.2$ & $18: 18: 20$ & 14.71 & IBL \\
\hline FIRST J1226.0+2604 & $12: 26: 04.1$ & $26: 04: 28$ & 16.29 & IBL \\
\hline RXS J1230.2+2517 & $12: 30: 14$ & $25: 18: 06$ & 14.9 & IBL \\
\hline $2 \mathrm{E} 1258+1437$ & $12: 31: 23.9$ & $14: 21: 25$ & 14.91 & IBL \\
\hline MS $1229.2+6430$ & $12: 31: 31.3$ & $64: 14: 18$ & 16.25 & IBL \\
\hline B2 $1229+29$ & $12: 31: 43.6$ & $28: 47: 49$ & - & - \\
\hline FIRST J1236.3+3900 & $12: 36: 23.1$ & 39:00:01 & 16.61 & HBL \\
\hline MS $1235.4+6315$ & $12: 37: 38.6$ & $62: 58: 44$ & 15.98 & IBL \\
\hline RXS J1237.0+3020 & $12: 37: 05.5$ & 30:20:04 & - & - \\
\hline 1ES $1239+069$ & $12: 41: 48.3$ & $06: 36: 01$ & 17.38 & HBL \\
\hline RXS J1241.6+3440 & $12: 41: 41.2$ & $34: 40: 32$ & - & - \\
\hline Ton 116 & $12: 43: 12.7$ & $36: 27: 44$ & - & - \\
\hline
\end{tabular}


Table 3. continued.

\begin{tabular}{|c|c|c|c|c|}
\hline Source & $\overline{R A(J 2000)}$ & $\operatorname{Dec}(\mathrm{J} 2000)$ & $\log v_{\text {peak }}$ & Class \\
\hline PG 1246+586 & $12: 48: 18.8$ & $58: 20: 29$ & 15.05 & IBL \\
\hline 1ES 1249+174E & $12: 51: 45.5$ & $17: 11: 17$ & - & - \\
\hline FIRST J1252.3+2640 & $12: 52: 19.5$ & $26: 40: 53$ & - & - \\
\hline S4 $1250+53$ & $12: 53: 11.9$ & $53: 01: 11$ & 14.82 & IBL \\
\hline 1ES $1255+244$ & $12: 57: 31.9$ & $24: 12: 40$ & 16.89 & HBL \\
\hline MS $1256.3+0151$ & $12: 58: 54.6$ & $01: 34: 43$ & - & - \\
\hline MS 1258.4+6401 & 13:00:17.6 & $63: 44: 39$ & 16.35 & IBL \\
\hline FIRST J1301.7+4056 & $13: 01: 45.7$ & $40: 56: 24$ & 16.55 & HBL \\
\hline RXS J1302.9+5056 & $13: 02: 55.5$ & $50: 56: 17$ & - & - \\
\hline MC2 1307+12 & 13:09:33.9 & $11: 54: 24$ & 13.07 & LBL \\
\hline 1WGA J1309.6+0828 & 13:09:38.9 & $08: 28: 28$ & 14.64 & IBL \\
\hline AUCVn & $13: 10: 28.66$ & $32: 30: 43.8$ & 13.75 & LBL \\
\hline HS $1309+2605$ & $13: 12: 19.2$ & $25: 49: 58$ & - & - \\
\hline TEX $1312+240$ & $13: 14: 43.8$ & $23: 48: 26$ & 15.84 & IBL \\
\hline RXS J1319.5+1405 & $13: 19: 31.7$ & $14: 05: 34$ & 20.85 & HBL \\
\hline UM 566 & 13:19:55.1 & $01: 52: 58$ & - & - \\
\hline $1 \mathrm{ES} 1320+084 \mathrm{~N}$ & $13: 22: 54.9$ & $08: 10: 10$ & 13.12 & LBL \\
\hline RXS J1324.0+5739 & $13: 24: 00.8$ & $57: 39: 16$ & 15.48 & IBL \\
\hline RXS J1326.2+2933 & $13: 26: 15$ & $29: 33: 29$ & - & - \\
\hline RXS J1326.2+1230 & $13: 26: 17.6$ & $12: 30: 00$ & 16.32 & IBL \\
\hline RX J1340.1+2743 & $13: 40: 10.9$ & $27: 43: 48$ & - & - \\
\hline RXS J1340.4+4410 & $13: 40: 29.8$ & $44: 10: 04$ & 16.51 & HBL \\
\hline RXS J1341.0+3959 & $13: 41: 05$ & $39: 59: 45$ & 20.06 & HBL \\
\hline $1338.8+2705$ & $13: 41: 05.8$ & $26: 50: 26$ & - & - \\
\hline $1340.8+2721$ & $13: 43: 05.1$ & $27: 06: 24$ & - & - \\
\hline RXS J1353.4+5601 & $13: 53: 28$ & $56: 00: 55$ & 19.23 & HBL \\
\hline FIRST J1354.4+3706 & $13: 54: 26.7$ & $37: 06: 54$ & 16.92 & HBL \\
\hline RXS J1359.8+5911 & $13: 59: 53.7$ & $59: 11: 01$ & 13.66 & LBL \\
\hline MC $1400+162$ & $14: 02: 44.5$ & $15: 59: 57$ & 16.47 & IBL \\
\hline RXS J1404.8+6554 & 14:04:49.6 & $65: 54: 30$ & - & - \\
\hline MS 1402.3+0416 & 14:04:51 & 04:02:02 & 15.83 & IBL \\
\hline MS $1407.9+5954$ & $14: 09: 23.5$ & $59: 39: 41$ & 16.63 & HBL \\
\hline PKS 1407+022 & $14: 10: 04.6$ & 02:03:07 & 13.69 & LBL \\
\hline RXS J1410.5+6100 & $14: 10: 31.7$ & 61:00:10 & 20.25 & HBL \\
\hline FIRST J1414.1+3430 & $14: 14: 09.3$ & $34: 30: 57$ & - & - \\
\hline RGB J1415+485 & $14: 15: 36.8$ & $48: 30: 30$ & 14.56 & IBL \\
\hline PKS 1413+135 & $14: 15: 58.8$ & $13: 20: 24$ & 12.83 & LBL \\
\hline CRSS $1416.3+1137$ & $14: 16: 20.7$ & $11: 37: 37$ & - & - \\
\hline $2 \mathrm{E} 1415+2557$ & $14: 17: 56.6$ & $25: 43: 25$ & 19.24 & $\mathrm{HBL}$ \\
\hline OQ 530 & $14: 19: 46.6$ & $54: 23: 14$ & 14.16 & LBL \\
\hline RXS J1422.6+5801 & $14: 22: 39$ & $58: 01: 55$ & - & - \\
\hline FIRST J1426.1+3404 & $14: 26: 07.7$ & $34: 04: 26$ & 14.1 & LBL \\
\hline PKS $1424+240$ & $14: 27: 00.5$ & $23: 48: 00$ & 15.7 & IBL \\
\hline RGB J1427+541 & $14: 27: 30.3$ & $54: 09: 24$ & 14.89 & IBL \\
\hline H $1426+428$ & $14: 28: 32.7$ & $42: 40: 21$ & 18.55 & HBL \\
\hline TEX $1428+370$ & $14: 30: 40.6$ & $36: 49: 03$ & 14.26 & LBL \\
\hline CSO 474 & $14: 36: 45.7$ & $35: 57: 01$ & - & - \\
\hline RXS J1436.9+5639 & $14: 36: 57.8$ & $56: 39: 25$ & 17.5 & HBL \\
\hline PG $1437+398$ & $14: 39: 17.5$ & $39: 32: 43$ & 16.7 & HBL \\
\hline 1ES $1440+122$ & $14: 42: 48.3$ & $12: 00: 40$ & 16.45 & IBL \\
\hline MS $1443.5+6349$ & $14: 44: 34.9$ & $63: 36: 06$ & 17.05 & HBL \\
\hline RXS J1445.0-0326 & $14: 45: 06.2$ & $-03: 26: 12$ & - & - \\
\hline RXS J1448.0+3608 & 14:48:00.6 & $36: 08: 31$ & 16.73 & HBL \\
\hline RXS J1449.5+2746 & $14: 49: 32.8$ & $27: 46: 21$ & 18.82 & HBL \\
\hline RXS J1451 4+6354 & $14: 51: 27.5$ & $63: 54: 19$ & - & - \\
\hline
\end{tabular}


Table 3. continued.

\begin{tabular}{|c|c|c|c|c|}
\hline Source & $\overline{\mathrm{RA}(\mathrm{J} 2000)}$ & $\overline{\operatorname{Dec}(\mathbf{J} 2000)}$ & $\log v_{\text {peak }}$ & Class \\
\hline SBS $1452+516$ & $14: 54: 27.1$ & $51: 24: 33$ & - & - \\
\hline RXS J1456.0+5048 & $14: 56: 03.7$ & $50: 48: 25$ & 19.94 & HBL \\
\hline RXS J1458.4+4832 & $14: 58: 28$ & $48: 32: 40$ & 21.46 & HBL \\
\hline B3 $1456+375$ & $14: 58: 44.8$ & $37: 20: 22$ & 13.47 & LBL \\
\hline MS $1458.8+2249$ & 15:01:01.9 & 22:38:06 & 15.26 & IBL \\
\hline FIRST J1502.1+2528 & $15: 02: 08.3$ & $25: 28: 45$ & 14.17 & LBL \\
\hline FIRST J1502.5+3350 & $15: 02: 34$ & $33: 50: 55$ & 13.66 & LBL \\
\hline RXS J1508.7+2709 & $15: 08: 42.9$ & 27:09:10 & 17.3 & HBL \\
\hline SBS $1508+561$ & $15: 09: 48$ & $55: 56: 17$ & 15.22 & IBL \\
\hline FIRST J1515.9+2426 & $15: 15: 56.2$ & $24: 26: 20$ & 15.89 & IBL \\
\hline RXS J1516.7+2918 & $15: 16: 41.6$ & $29: 18: 10$ & 18.62 & HBL \\
\hline PKS 1514+197 & $15: 16: 56.8$ & $19: 32: 12$ & 13.6 & LBL \\
\hline $1 \mathrm{H} 1515+660$ & $15: 17: 47.6$ & $65: 25: 24$ & 18.11 & HBL \\
\hline FIRST J1530.7+2310 & $15: 30: 44$ & $23: 10: 13$ & - & - \\
\hline FAQS J1530.7+5329 & $15: 30: 44.5$ & $53: 29: 28$ & 16.88 & HBL \\
\hline RXS J1532.0+3016 & $15: 32: 02.2$ & $30: 16: 29$ & 17.05 & HBL \\
\hline RXS J1533.4+3416 & $15: 33: 24.3$ & $34: 16: 41$ & 18.32 & HBL \\
\hline RGB J1534+372 & $15: 34: 47.2$ & $37: 15: 55$ & 14.26 & LBL \\
\hline 1ES $1533+535$ & $15: 35: 00.8$ & $53: 20: 37$ & 19.68 & HBL \\
\hline FIRST J1535.4+3922 & $15: 35: 29.1$ & $39: 22: 46$ & 16.38 & IBL \\
\hline MS 1534.2+0148 & $15: 36: 46.8$ & 01:37:59 & 18.83 & HBL \\
\hline 1ES $1544+820$ & $15: 40: 15.8$ & $81: 55: 07$ & 17.79 & $\mathrm{HBL}$ \\
\hline 4C 14.6 & $15: 40: 46.5$ & $14: 47: 45.9$ & 14.85 & IBL \\
\hline RXS J1542.9+6129 & $15: 42: 56.9$ & $61: 29: 56$ & 14.72 & IBL \\
\hline RXS J1544.3+0458 & $15: 44: 18.7$ & $04: 58: 24$ & 16.77 & HBL \\
\hline MS 1552.1+2020 & $15: 54: 24.1$ & $20: 11: 25$ & 17.12 & HBL \\
\hline PG $1553+11$ & $15: 55: 43.1$ & $11: 11: 24$ & 16.49 & IBL \\
\hline MYC 1557+566 & $15: 58: 48.5$ & $56: 25: 14$ & - & - \\
\hline RXS J1602.2+3050 & $16: 02: 18$ & 30:51:09 & 16.42 & IBL \\
\hline PKS 1604+159 & $16: 07: 06.4$ & $15: 51: 34$ & 14.73 & IBL \\
\hline RXS J1610.0+6710 & 16:10:04.1 & $67: 10: 26$ & 17.45 & HBL \\
\hline B3 $1615+412$ & $16: 17: 06.6$ & 41:06:45 & 14.41 & LBL \\
\hline 87GB 16166+2206 & $16: 18: 47.9$ & $21: 59: 26$ & 15.66 & IBL \\
\hline 4C 37.46 & $16: 21: 11.3$ & $37: 46: 04.9$ & - & - \\
\hline RXJ 1624.7+3726 & $16: 24: 43.4$ & $37: 26: 42$ & - & - \\
\hline RXS J1624.9+7554 & $16: 24: 56.5$ & $75: 54: 55$ & 13.3 & LBL \\
\hline RXS J1626.4+3513 & $16: 26: 25.6$ & $35: 13: 38$ & 15.28 & IBL \\
\hline RXS J1631.3+4217 & $16: 31: 24.7$ & 42:17:03 & 18.99 & HBL \\
\hline RXS J1638.0+7326 & $16: 38: 02.6$ & $73: 26: 10$ & - & - \\
\hline RXS J1644.2+4546 & $16: 44: 20$ & $45: 46: 44$ & 17.48 & HBL \\
\hline $1643.2+4021$ & $16: 44: 53.2$ & $40: 16: 28$ & - & - \\
\hline FIRST J1645.9+2947 & $16: 45: 57.7$ & $29: 47: 30$ & - & - \\
\hline FIRST J1651.1+4212 & $16: 51: 09.2$ & $42: 12: 53$ & 17.82 & HBL \\
\hline RXS J1651.6+7218 & $16: 51: 41.5$ & $72: 18: 19$ & - & - \\
\hline RGB J1652+403 & $16: 52: 50$ & $40: 23: 10$ & 14.97 & IBL \\
\hline MRK 501 & $16: 53: 52.2$ & $39: 45: 36$ & 16.84 & HBL \\
\hline B3 $1659+399$ & $17: 01: 24.6$ & $39: 54: 36$ & 13.41 & LBL \\
\hline FIRST J1702.1+2643 & 17:02:09.6 & $26: 43: 15$ & 14.27 & LBL \\
\hline FIRST J1702.6+3115 & $17: 02: 38.6$ & $31: 15: 43$ & 13.49 & LBL \\
\hline RXS J1704.8+7138 & $17: 04: 46.9$ & $71: 38: 18$ & 15.58 & IBL \\
\hline MS 1704.9+6046 & $17: 05: 34.9$ & $60: 42: 17$ & 17.17 & HBL \\
\hline FIRST J1712.8+2931 & $17: 12: 48.8$ & $29: 31: 17$ & 18.79 & HBL \\
\hline RXS J1718.6+7358 & $17: 18: 40.1$ & $73: 58: 15$ & - & - \\
\hline PKS 1717+177 & $17: 19: 13.1$ & $17: 45: 07$ & 13.08 & LBL \\
\hline RXS J1719.6+7443 & $17: 19: 41$ & $74: 43: 00$ & 14.19 & LBL \\
\hline
\end{tabular}


Table 3. continued.

\begin{tabular}{|c|c|c|c|c|}
\hline Source & $\overline{R A(J 2000)}$ & $\operatorname{Dec}(\mathrm{J} 2000)$ & $\log v_{\text {peak }}$ & Class \\
\hline B2 1722+40 & $17: 24: 05.5$ & $40: 04: 38$ & 13 & $\overline{L B L}$ \\
\hline H $1722+119$ & $17: 25: 04.4$ & $11: 52: 16$ & 15.8 & IBL \\
\hline IZw187 & $17: 28: 18.6$ & $50: 13: 11$ & 17.42 & $\mathrm{HBL}$ \\
\hline RXS J1728.6+7041 & $17: 28: 38.3$ & $70: 41: 08$ & 13.6 & LBL \\
\hline RXS J1732.0+6926 & $17: 32: 05.4$ & $69: 26: 16$ & - & - \\
\hline OT 465 & $17: 39: 57.1$ & $47: 37: 59$ & 13.91 & LBL \\
\hline RGBJ $1742+597$ & $17: 42: 32$ & $59: 45: 06$ & 14.18 & LBL \\
\hline NPM1G + 19.0510 & $17: 43: 57.9$ & $19: 35: 09$ & 17.91 & HBL \\
\hline B3 $1743+398 B$ & $17: 45: 37.7$ & $39: 51: 31$ & 17.71 & $\mathrm{HBL}$ \\
\hline 1ES $1745+504$ & $17: 46: 32.3$ & $50: 28: 09$ & - & - \\
\hline B3 $1746+470$ & $17: 47: 26.6$ & $46: 58: 51$ & 13.96 & LBL \\
\hline S4 1749+70 & $17: 48: 33.1$ & $70: 05: 50$ & 14.55 & IBL \\
\hline B3 $1747+433$ & $17: 49: 00.4$ & $43: 21: 52$ & 13.56 & LBL \\
\hline RXS J1750.0+4700 & 17:50:05 & $47: 00: 44$ & 18.1 & HBL \\
\hline PKS 1749+096 & $17: 51: 32.7$ & 09:39:01 & 12.78 & LBL \\
\hline RXS J1756.2+5522 & $17: 56: 15.9$ & $55: 22: 18$ & 19.9 & $\mathrm{HBL}$ \\
\hline MS 1757.7+7034 & $17: 57: 13.3$ & $70: 33: 37$ & 13.7 & LBL \\
\hline S5 $1803+784$ & 18:00:45.4 & $78: 28: 04$ & 14.05 & LBL \\
\hline RX J1801.7+6638 & $18: 01: 46.7$ & $66: 38: 40$ & - & - \\
\hline 3C 371 & 18:06:50.7 & $69: 49: 28$ & 14.65 & IBL \\
\hline RGB J1808+468 & 18:08:01.2 & $46: 49: 41$ & 14.63 & IBL \\
\hline RGB J1811+442 & $18: 11: 53.5$ & $44: 16: 29$ & 15.07 & IBL \\
\hline B2 $1811+31$ & $18: 13: 35.3$ & $31: 44: 17$ & 15.53 & IBL \\
\hline $4 C 56.27$ & $18: 24: 07.07$ & $56: 51: 01.5$ & 12.95 & LBL \\
\hline RXS J1829.4+5402 & $18: 29: 24.3$ & 54:03:00 & 15.07 & IBL \\
\hline Q $1832+6845$ & $18: 32: 36.6$ & $68: 48: 09$ & - & - \\
\hline RXS J1838.7+4802 & $18: 38: 49.2$ & $48: 02: 34$ & 13.52 & LBL \\
\hline RGB J1841+591 & $18: 41: 20.3$ & 59:06:08 & 14.91 & IBL \\
\hline RXS J1848.7+4245 & $18: 48: 47.1$ & $42: 45: 39$ & - & - \\
\hline 1ES $1853+671$ & $18: 53: 52.1$ & $67: 13: 55$ & 16.64 & HBL \\
\hline 87 GB $19021+5536$ & 19:03:11.6 & $55: 40: 39$ & 14.5 & LBL \\
\hline S4 1926+61 & $19: 27: 30.4$ & $61: 17: 32$ & 13.44 & LBL \\
\hline RXS J1931.1+0937 & 19:31:09.5 & $09: 37: 13$ & - & - \\
\hline 1ES 1959+650 & 19:59:59.9 & $65: 08: 55$ & 18.03 & HBL \\
\hline S5 2007+77 & 20:05:31.1 & $77: 52: 43$ & 13.15 & LBL \\
\hline S5 2010+72 & 20:09:52.3 & $72: 29: 19$ & 13.64 & LBL \\
\hline PKS 2012-017 & $20: 15: 15.1$ & $-01: 37: 33$ & 14.68 & IBL \\
\hline S5 2023+76 & $20: 22: 35.6$ & $76: 11: 26$ & 14.1 & LBL \\
\hline PKS 2032+107 & $20: 35: 22.3$ & $10: 56: 06$ & 14 & LBL \\
\hline 1ES 2037+521 & $20: 39: 23.5$ & $52: 19: 50$ & 16.13 & IBL \\
\hline PKS 2047+039 & 20:50:06.2 & 04:07:49 & 13.83 & LBL \\
\hline S5 2051+74 & $20: 51: 33.8$ & $74: 41: 40$ & 18.75 & HBL \\
\hline PKS 2131-021 & $21: 34: 10.2$ & $-01: 53: 17$ & 12.76 & LBL \\
\hline MS 2143.4+0704 & $21: 45: 52.3$ & $07: 19: 27$ & 13.92 & LBL \\
\hline PKS 2149+17 & $21: 52: 24.8$ & $17: 34: 39$ & 13.85 & LBL \\
\hline BL LAC & $22: 02: 43.3$ & $42: 16: 39$ & 14.28 & LBL \\
\hline RXS J2209.3+1031 & 22:09:18.5 & $10: 31: 43$ & 13.36 & LBL \\
\hline RXS J2219.7+2120 & $22: 19: 45.3$ & $21: 20: 48$ & 17.61 & HBL \\
\hline RXS J2225.1+1136 & $22: 25: 11.2$ & $11: 36: 01$ & - & - \\
\hline PKS 2223-114 & $22: 25: 43.7$ & $-11: 13: 41$ & 14.65 & IBL \\
\hline $3 \mathrm{C} 446$ & $22: 25: 45.1$ & $-04: 56: 34$ & 13.37 & LBL \\
\hline S5 2229+69 & $22: 30: 35.6$ & $69: 46: 29$ & 13.09 & LBL \\
\hline RXS J2233.0+1335 & $22: 33: 00.9$ & $13: 35: 59$ & 16.61 & HBL \\
\hline RGB J2243+203 & $22: 43: 54.7$ & $20: 21: 04$ & 14.15 & LBL \\
\hline B3 2247+381 & $22: 50: 05.8$ & $38: 24: 37$ & 15.61 & IBL \\
\hline
\end{tabular}


E. Nieppola et al.: SEDs of BL Lacertae Objects, Online Material $p 9$

Table 3. continued.

\begin{tabular}{l|cccc}
\hline \hline Source & RA(J2000) & Dec(J2000) & $\log v_{\text {peak }}$ & Class \\
\hline PKS 2254+074 & $22: 57: 17.3$ & $07: 43: 12$ & 14.18 & LBL \\
RXS J2304.6+3705 & $23: 04: 36.6$ & $37: 05: 08$ & 21.01 & HBL \\
B3 2311+396A & $23: 13: 50.2$ & $40: 03: 03$ & - & - \\
Q J2319+161 & $23: 19: 43.4$ & $16: 11: 51$ & 15.48 & IBL \\
TEX 2320+343 & $23: 22: 44$ & $34: 36: 14$ & 16.73 & HBL \\
1ES 2321+419 & $23: 23: 54.1$ & $42: 11: 19$ & 13.26 & LBL \\
B3 2322+396 & $23: 25: 17.9$ & $39: 57: 37$ & 15.29 & IBL \\
1ES 2326+174 & $23: 29: 03.3$ & $17: 43: 30$ & 18.07 & HBL \\
Q J2338+212 & $23: 38: 56.4$ & $21: 24: 41$ & 17.62 & HBL \\
MS 2336.5+0517 & $23: 39: 07$ & $05: 34: 36$ & 14.91 & IBL \\
1ES 2344+514 & $23: 47: 04.8$ & $51: 42: 18$ & 16.4 & IBL \\
MS 2347.4+1924 & $23: 50: 01.7$ & $19: 41: 52$ & 15.8 & IBL \\
RXS J2350.3-059 & $23: 50: 17.9$ & $-05: 59: 28$ & - & - \\
TEX 2348+360 & $23: 50: 36.7$ & $36: 22: 11$ & 16.1 & IBL \\
PKS 2354-02 & $23: 57: 25.1$ & $-01: 52: 15$ & - & - \\
\hline
\end{tabular}


E. Nieppola et al.: SEDs of BL Lacertae Objects, Online Material $p 10$
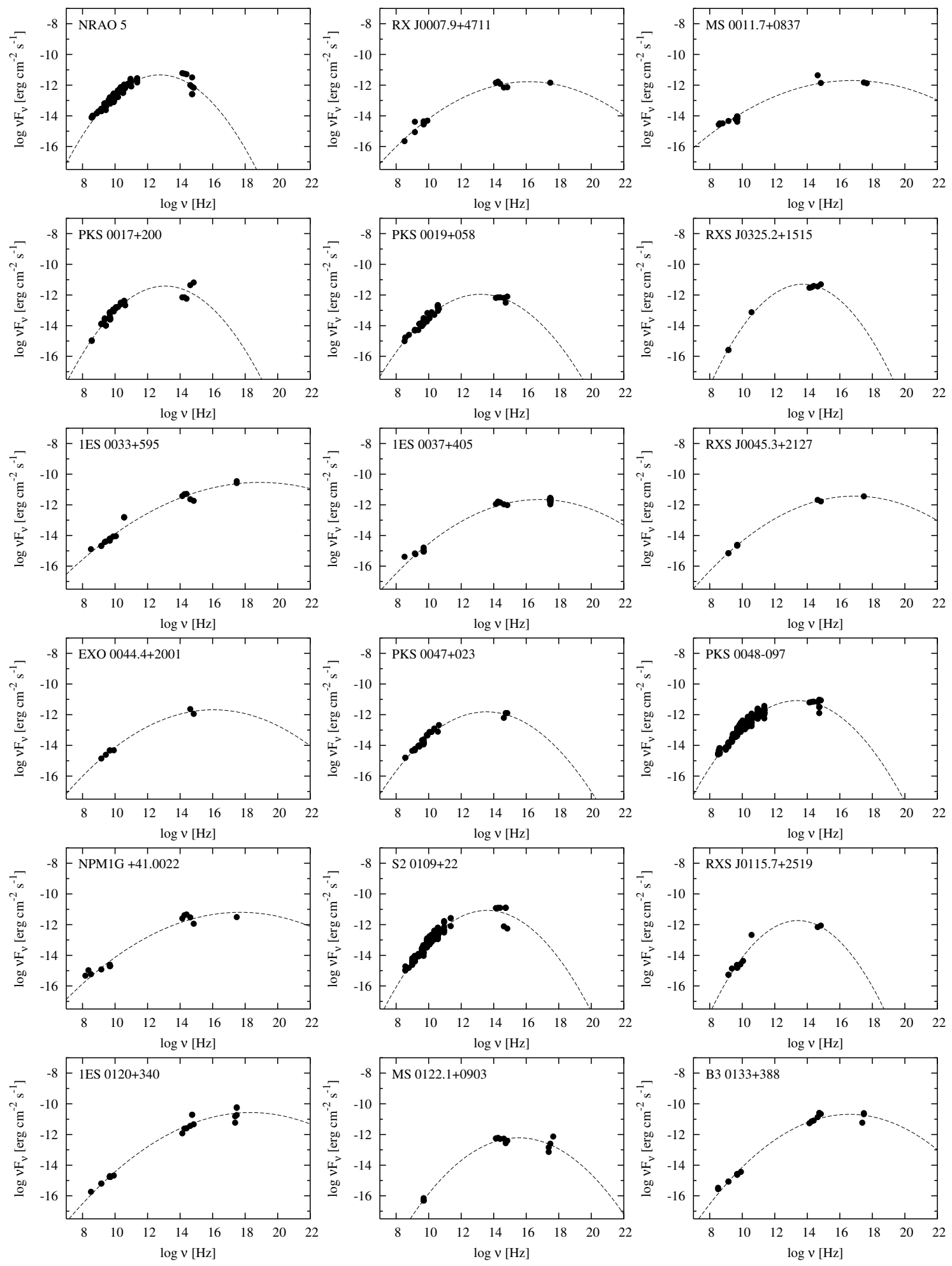

Fig. 13. Spectral energy distributions of the Metsähovi BL Lac sample. Only datapoints used in the fit are shown in the figure. 
E. Nieppola et al.: SEDs of BL Lacertae Objects, Online Material p 11
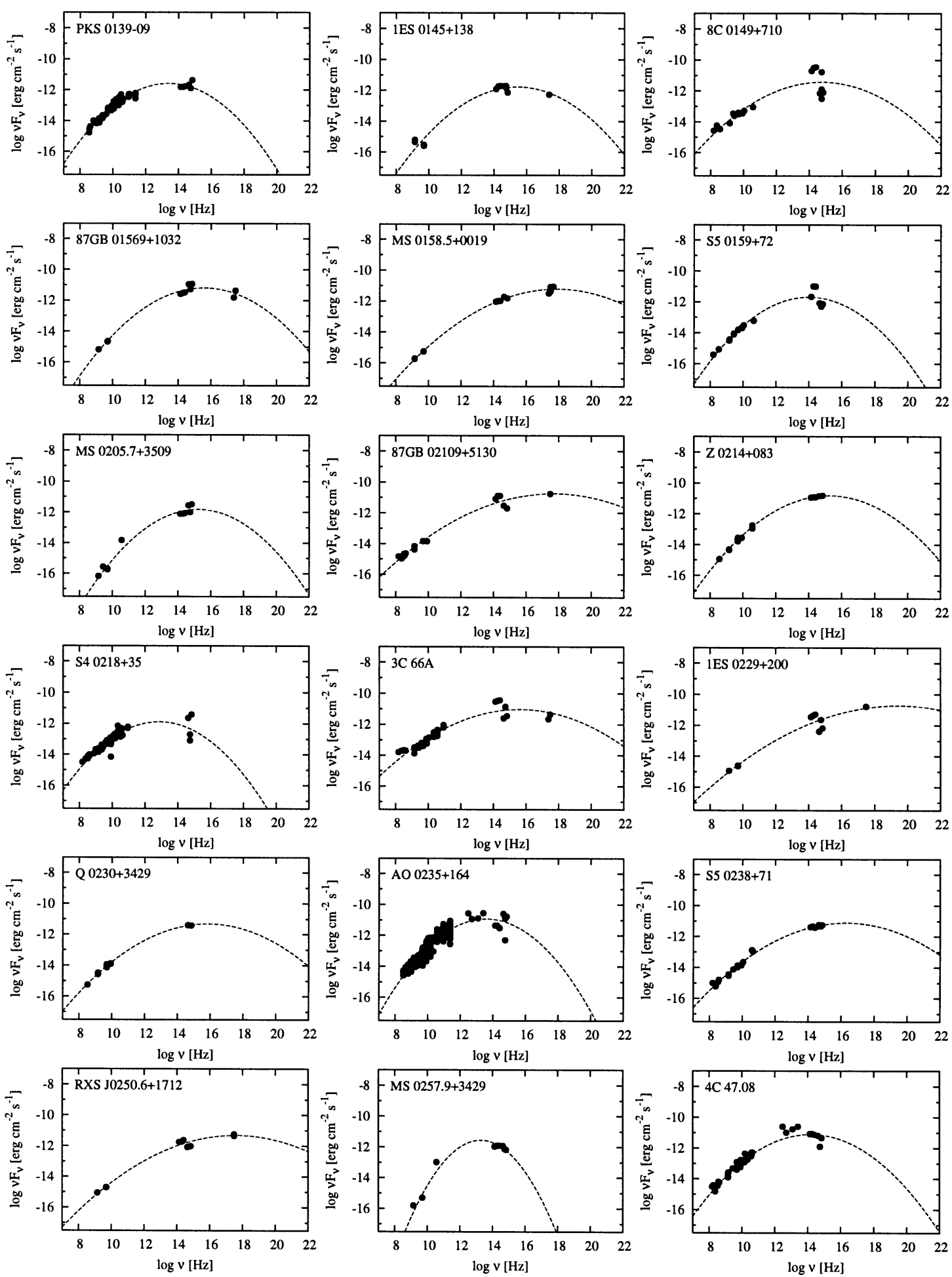

Fig. 13. continued. 
E. Nieppola et al.: SEDs of BL Lacertae Objects, Online Material p 12
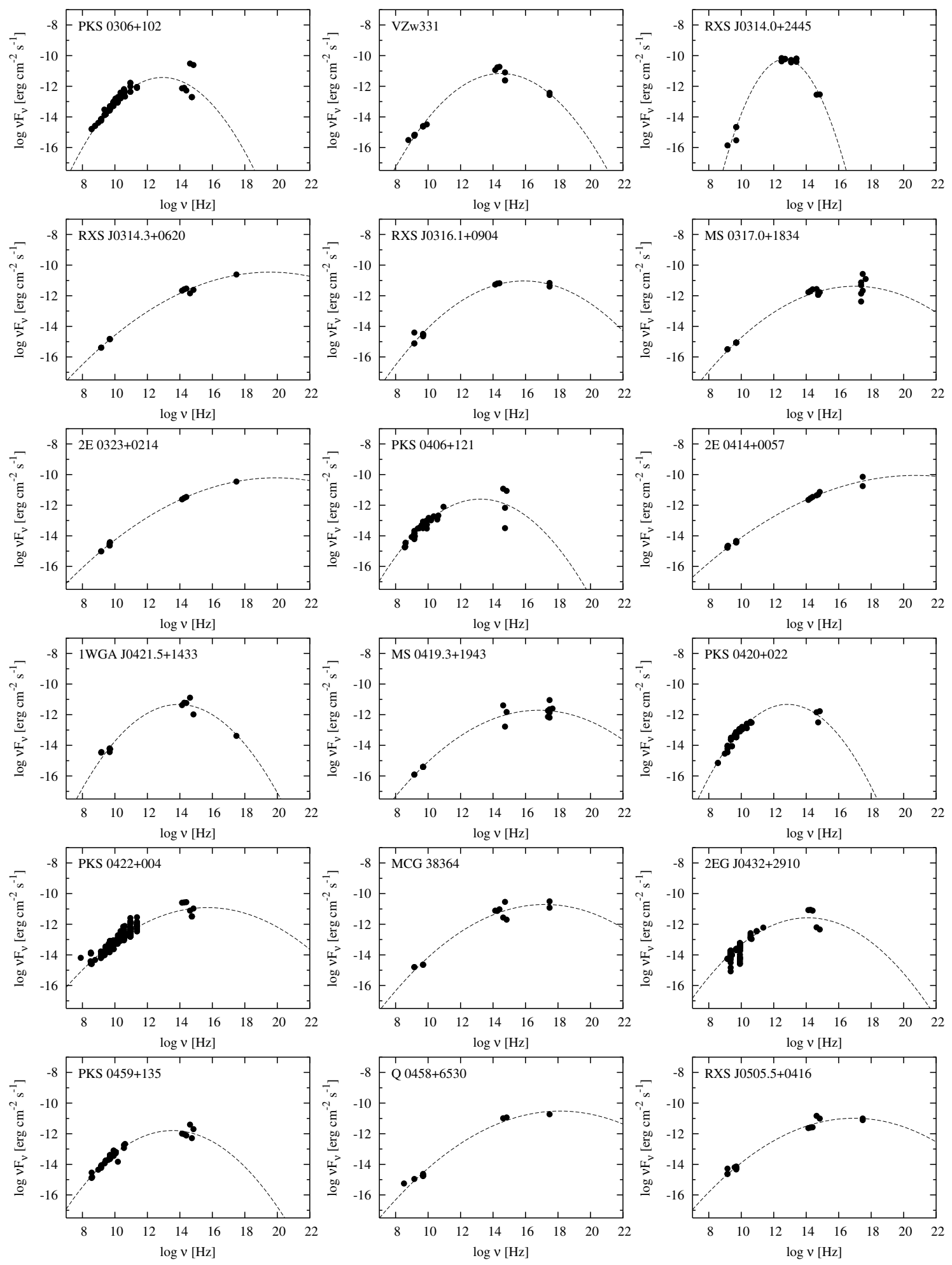

Fig. 13. continued. 
E. Nieppola et al.: SEDs of BL Lacertae Objects, Online Material p 13
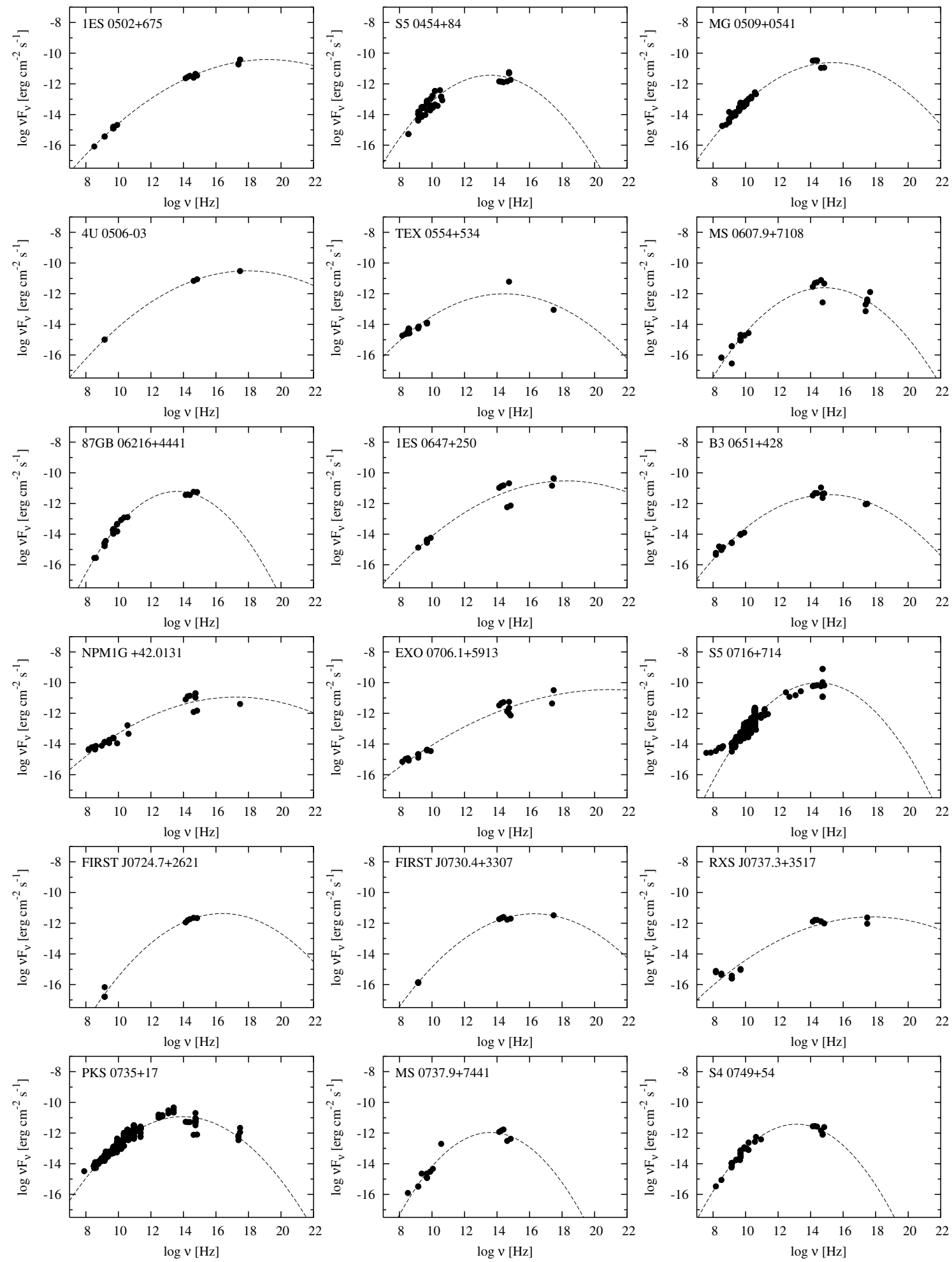

Fig. 13. continued. 
E. Nieppola et al.: SEDs of BL Lacertae Objects, Online Material p 14
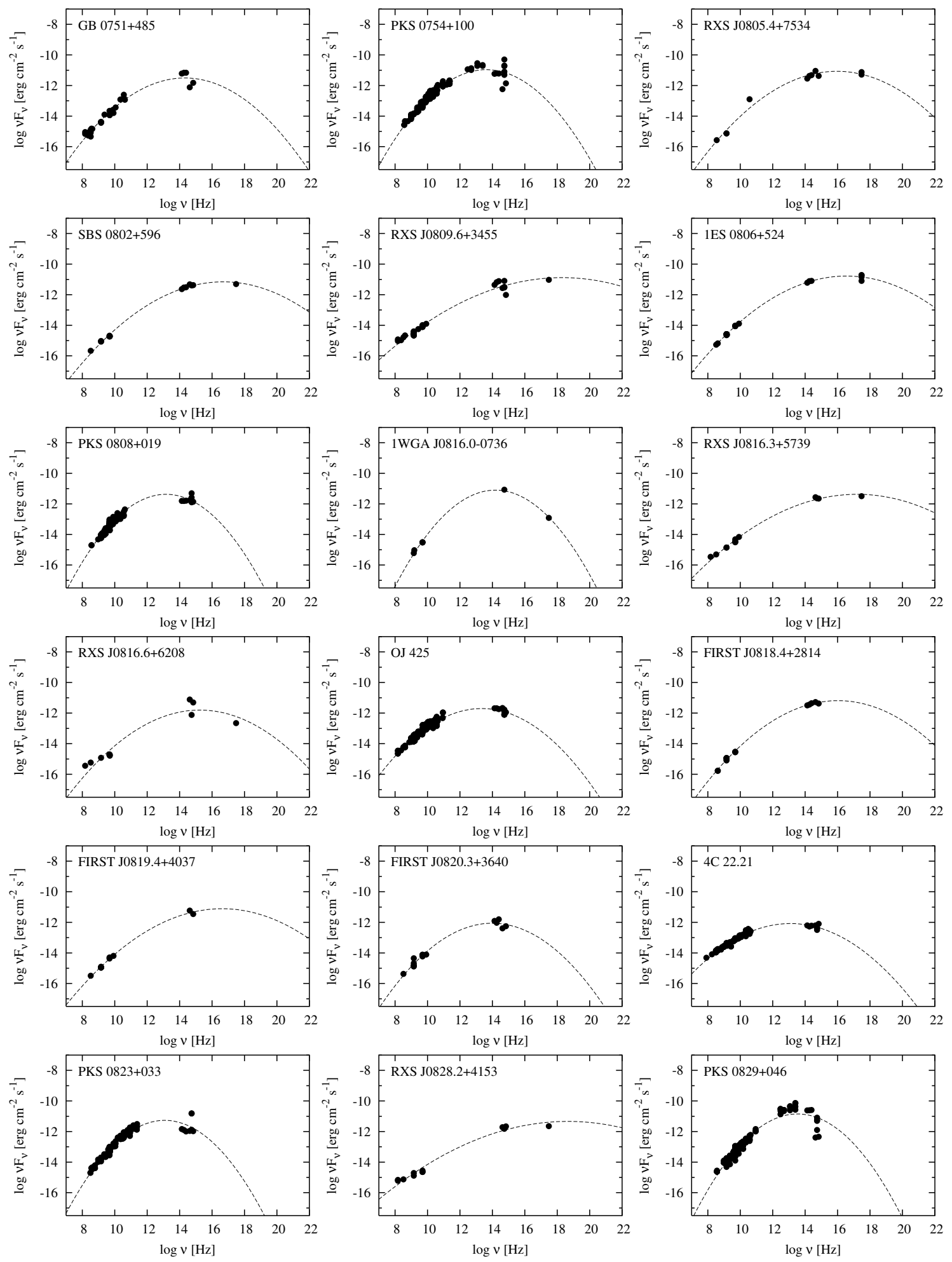

Fig. 13. continued. 
E. Nieppola et al.: SEDs of BL Lacertae Objects, Online Material p 15
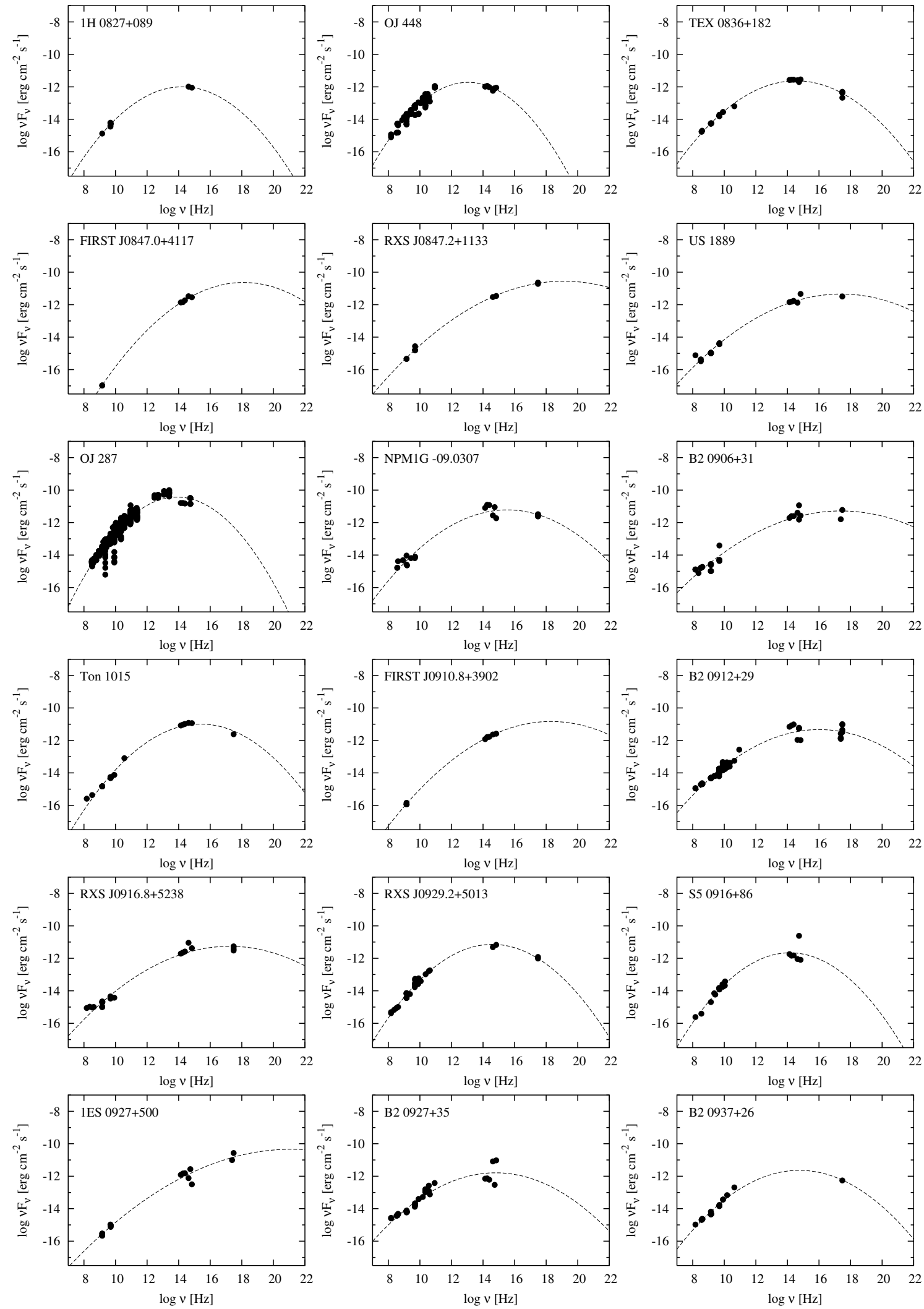

Fig. 13. continued. 
E. Nieppola et al.: SEDs of BL Lacertae Objects, Online Material p 16
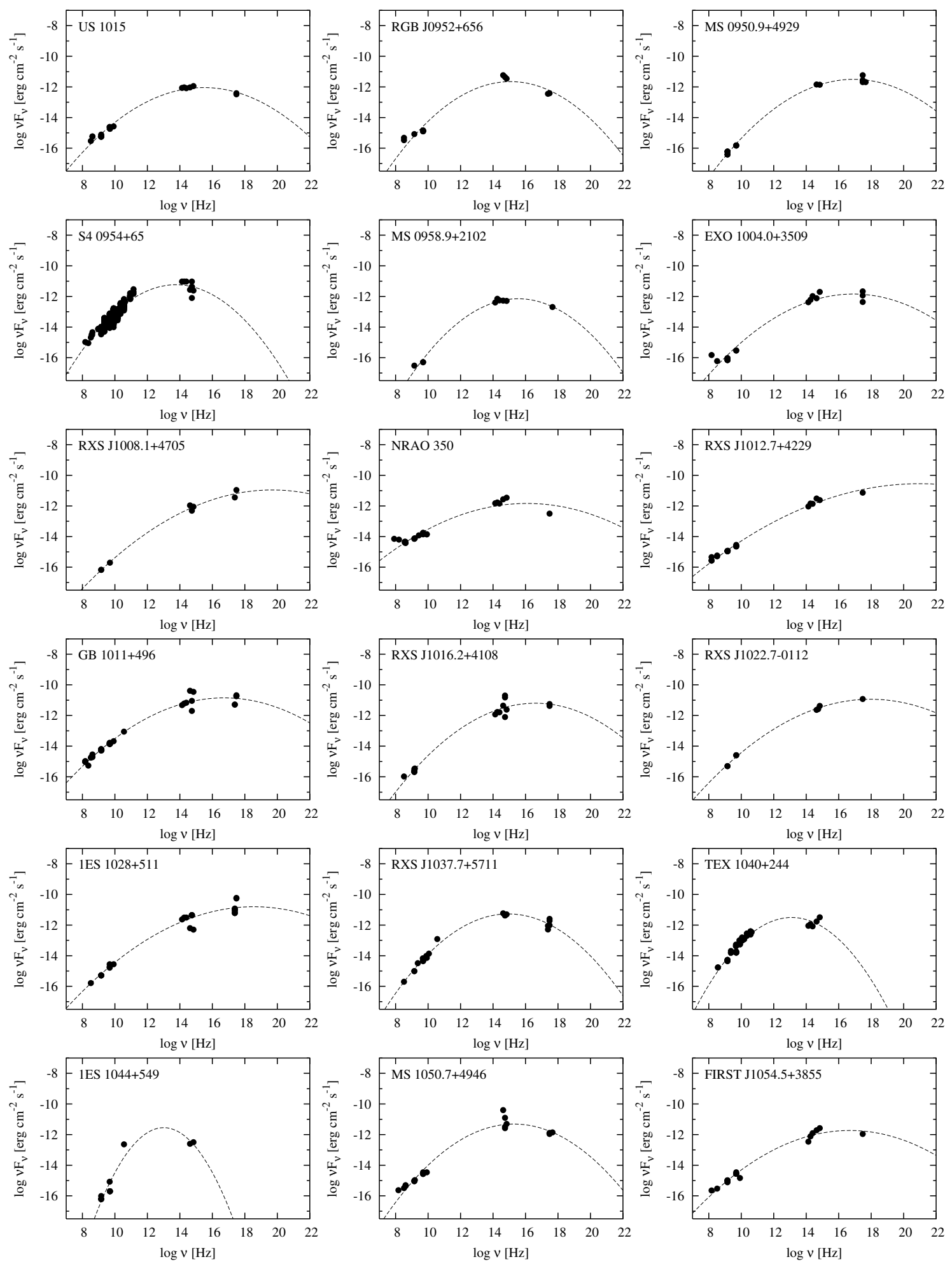

Fig. 13. continued. 
E. Nieppola et al.: SEDs of BL Lacertae Objects, Online Material p 17
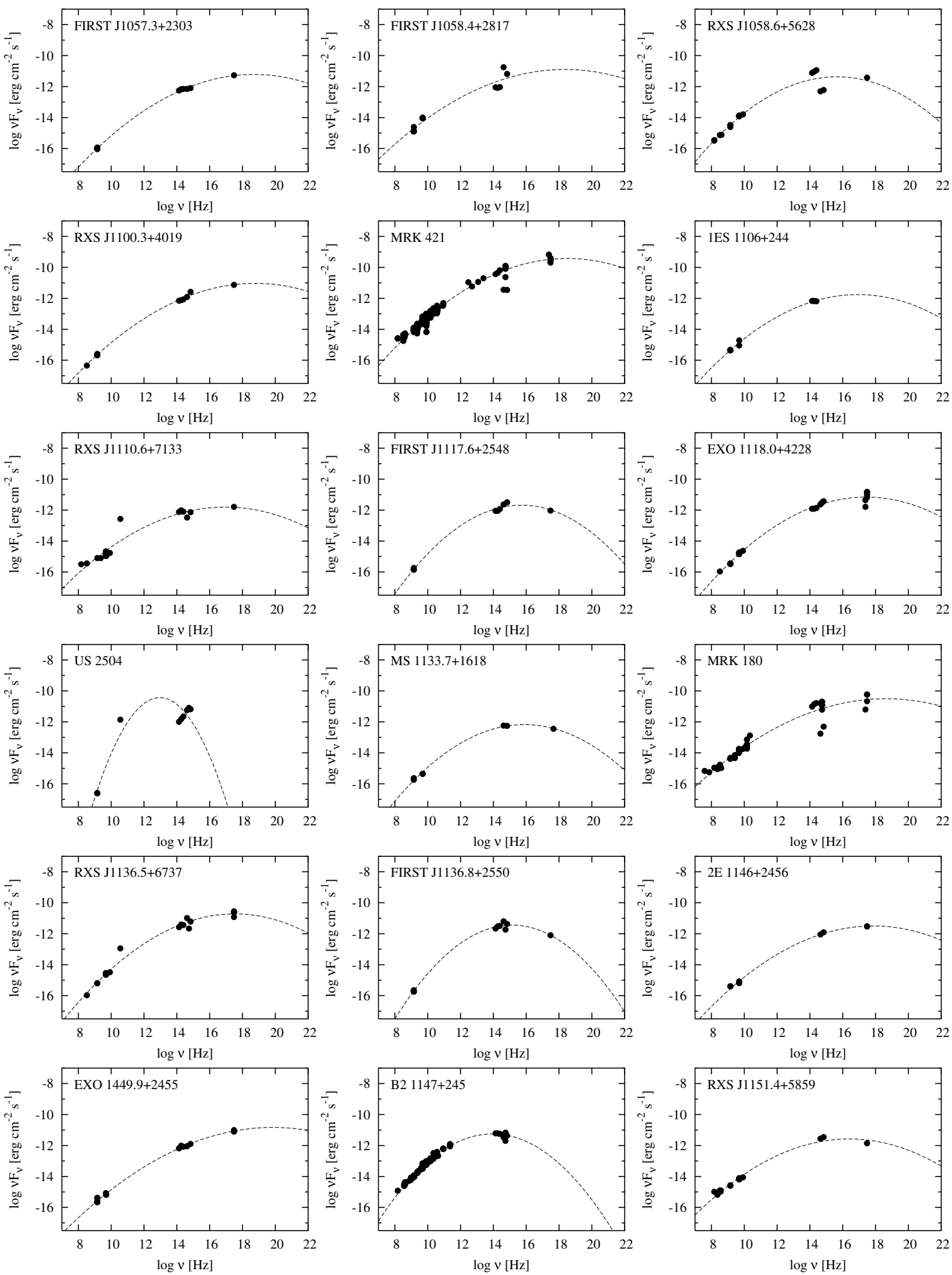

Fig. 13. continued. 
E. Nieppola et al.: SEDs of BL Lacertae Objects, Online Material p 18
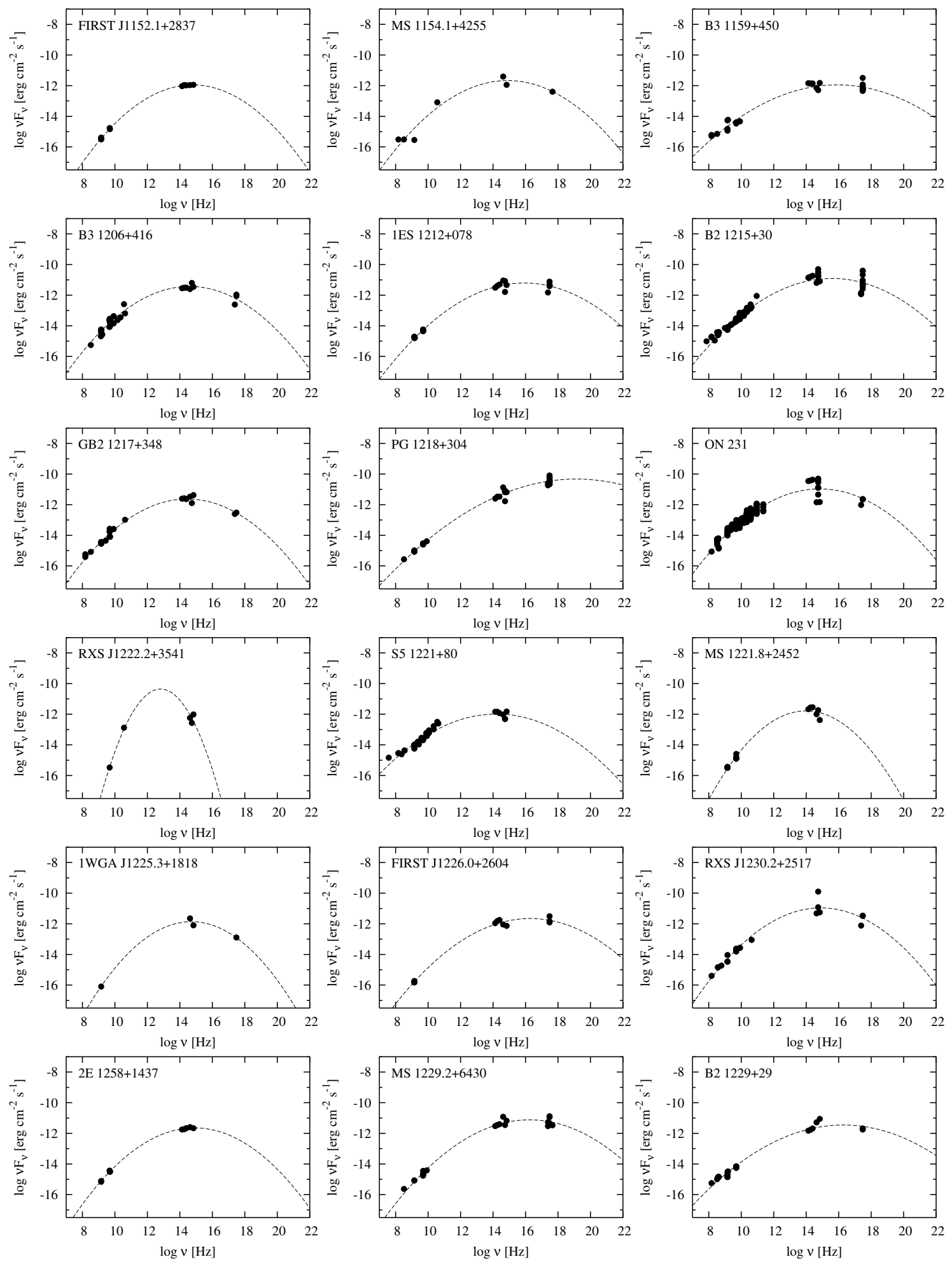

Fig. 13. continued. 
E. Nieppola et al.: SEDs of BL Lacertae Objects, Online Material p 19
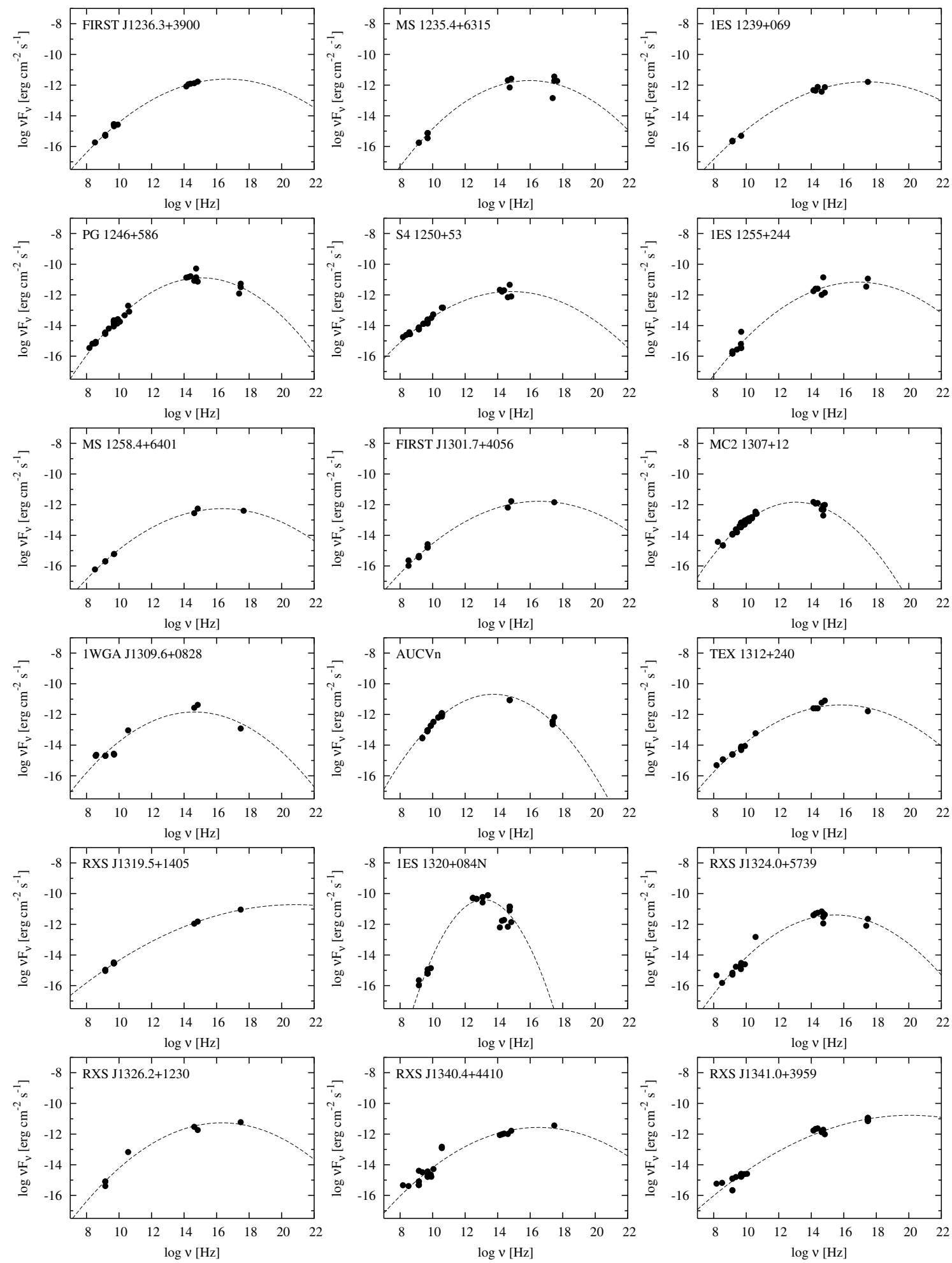

Fig. 13. continued. 
E. Nieppola et al.: SEDs of BL Lacertae Objects, Online Material p 20
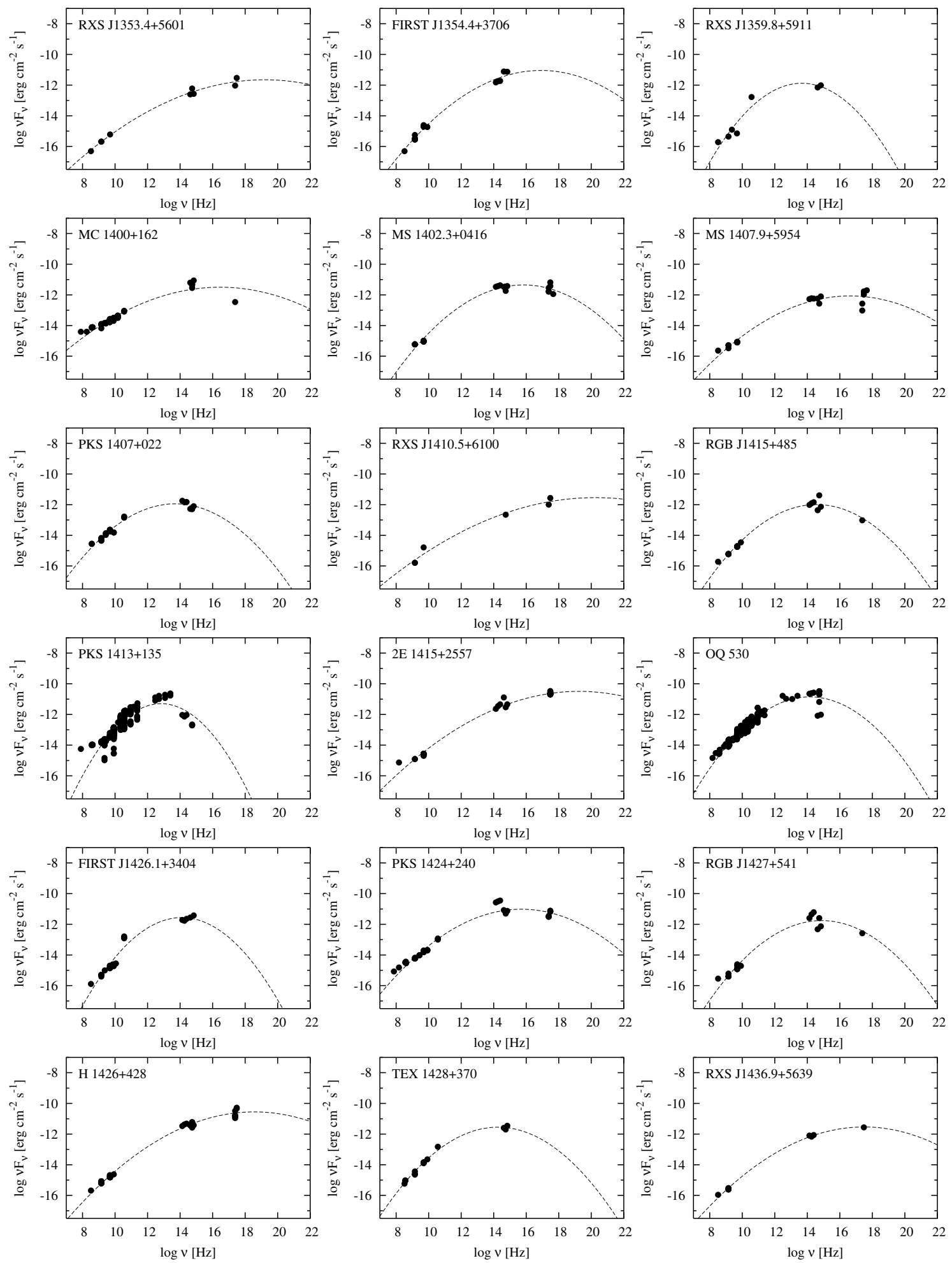

Fig. 13. continued. 
E. Nieppola et al.: SEDs of BL Lacertae Objects, Online Material p 21
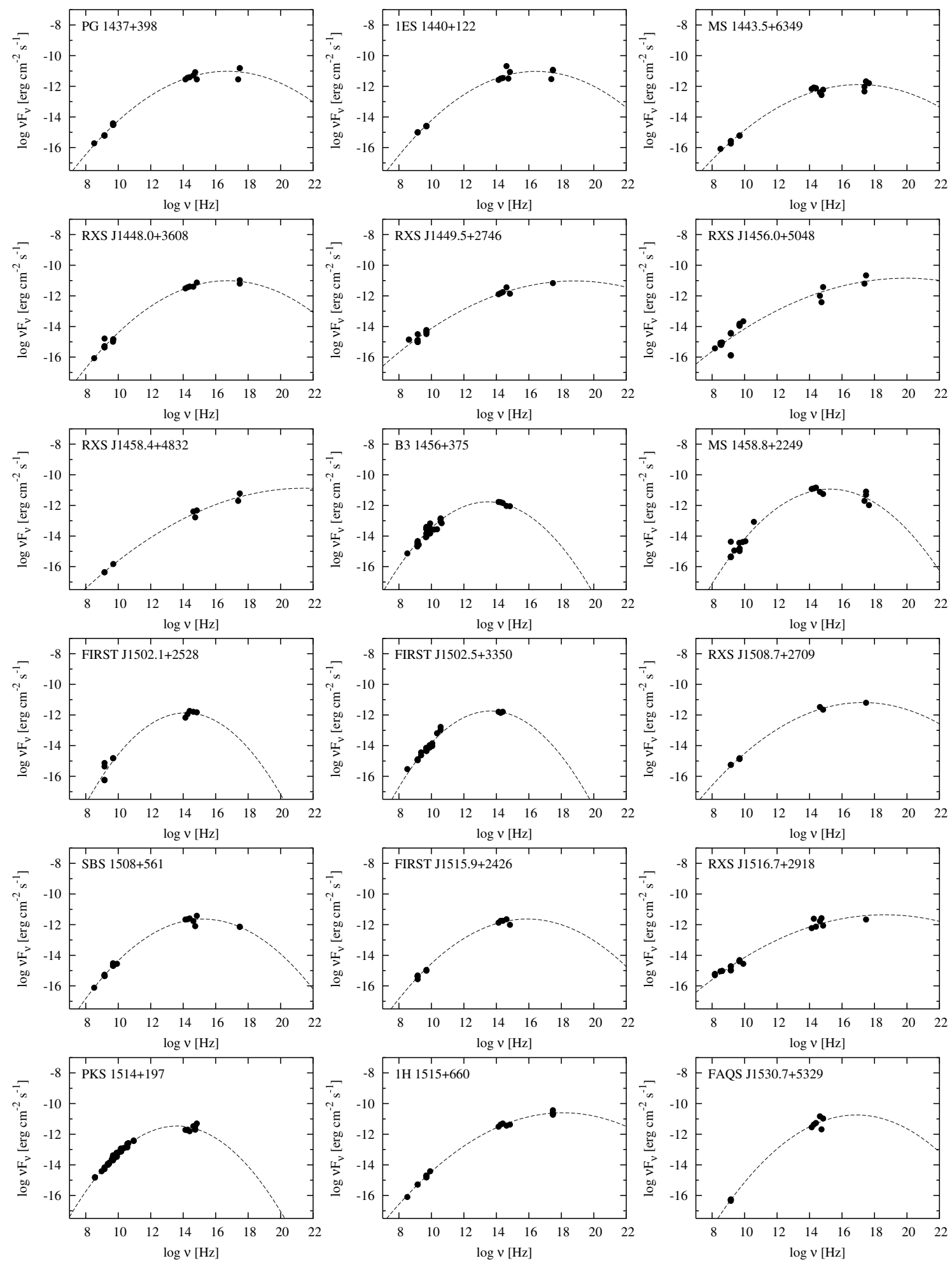

Fig. 13. continued. 
E. Nieppola et al.: SEDs of BL Lacertae Objects, Online Material p 22
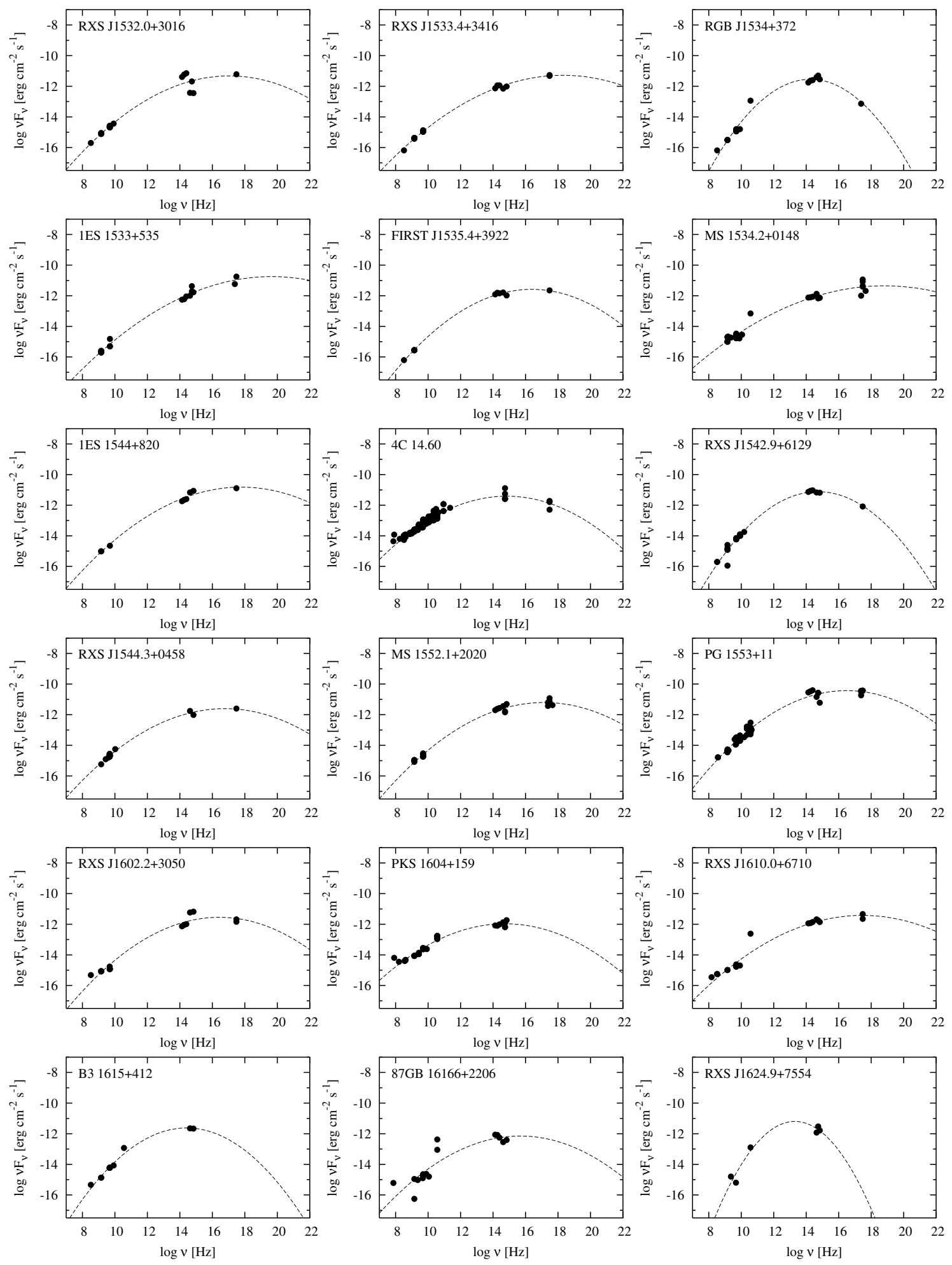

Fig. 13. continued. 
E. Nieppola et al.: SEDs of BL Lacertae Objects, Online Material p 23
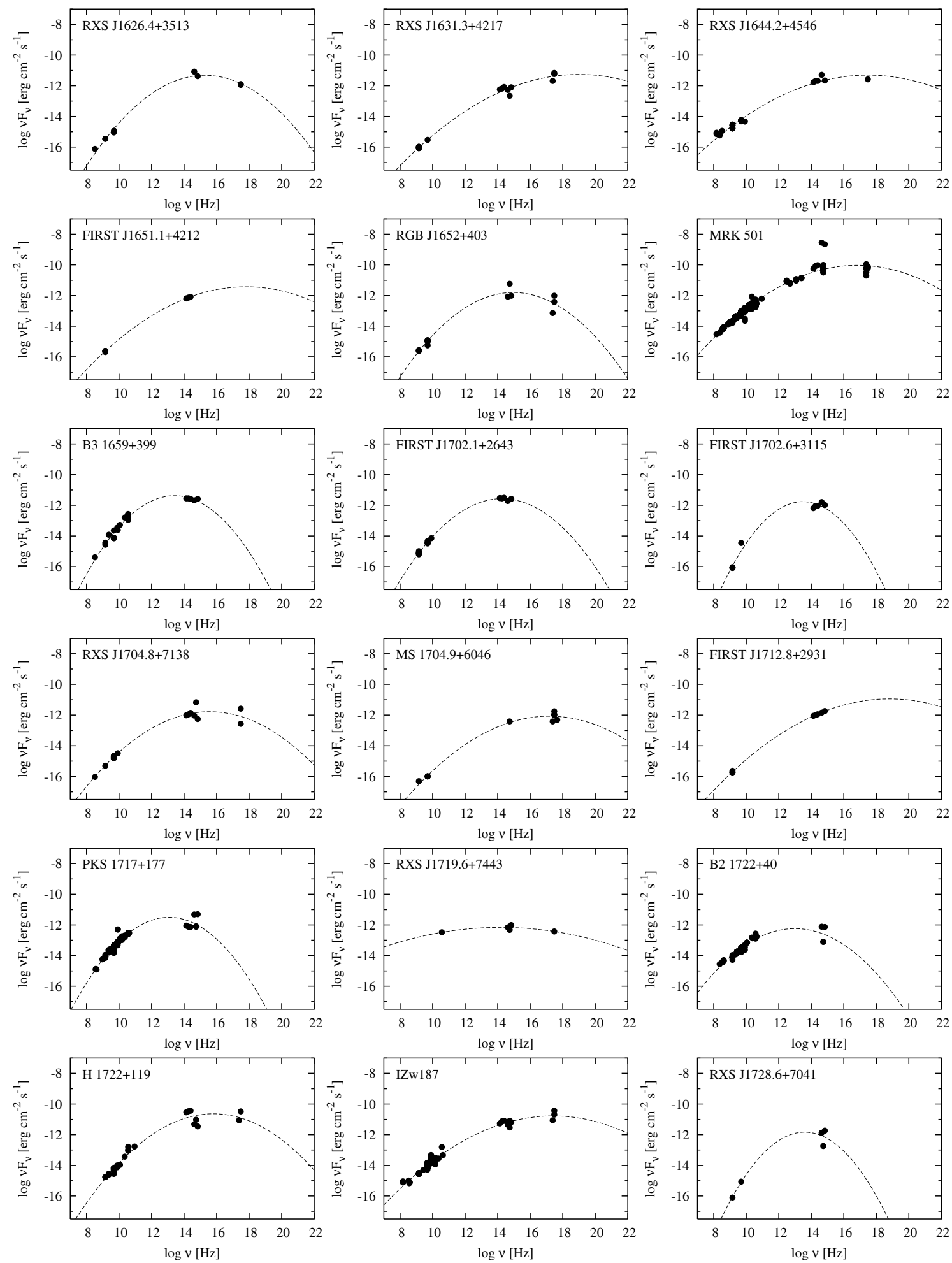

Fig. 13. continued. 
E. Nieppola et al.: SEDs of BL Lacertae Objects, Online Material p 24
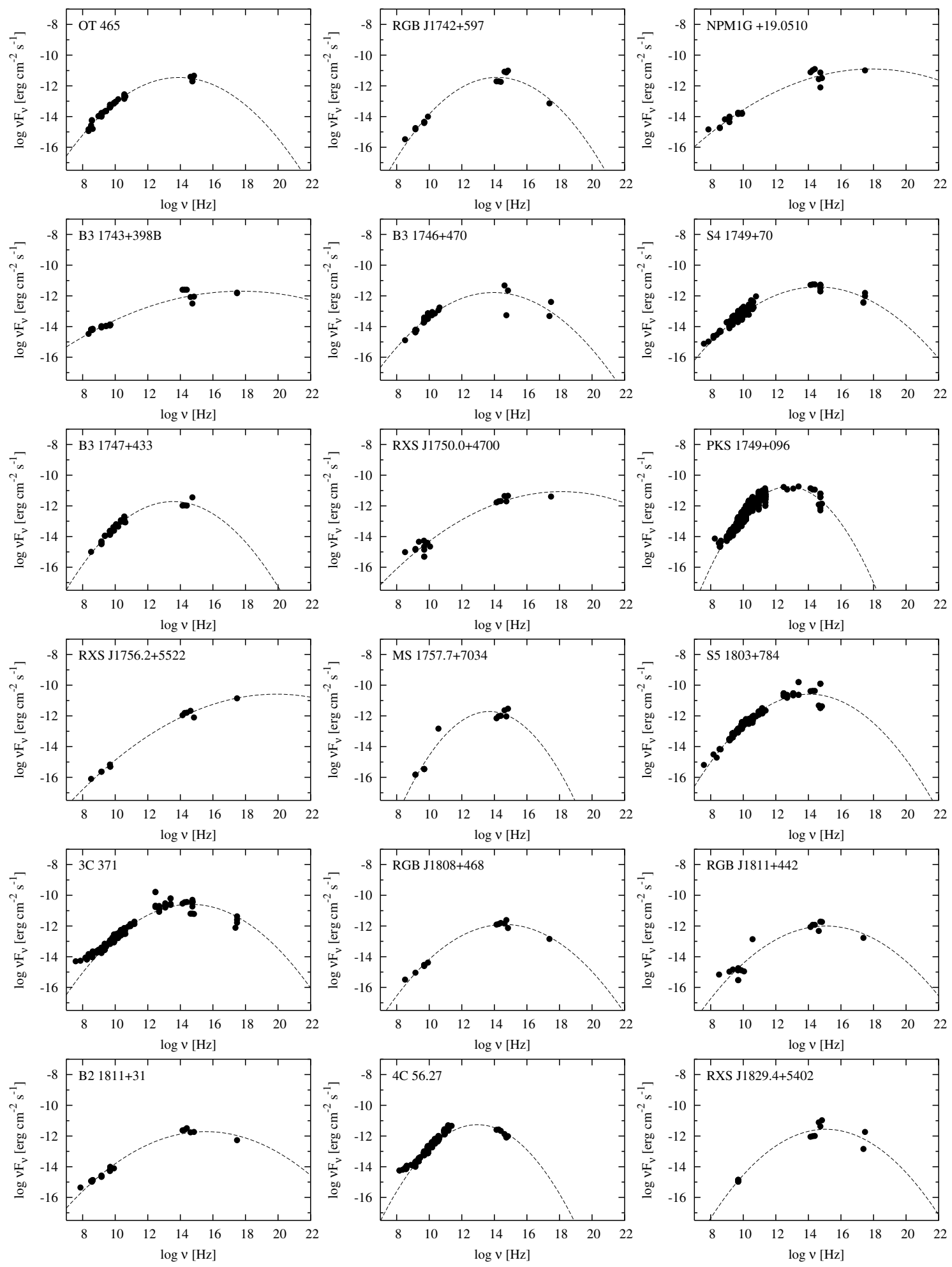

Fig. 13. continued. 
E. Nieppola et al.: SEDs of BL Lacertae Objects, Online Material p 25
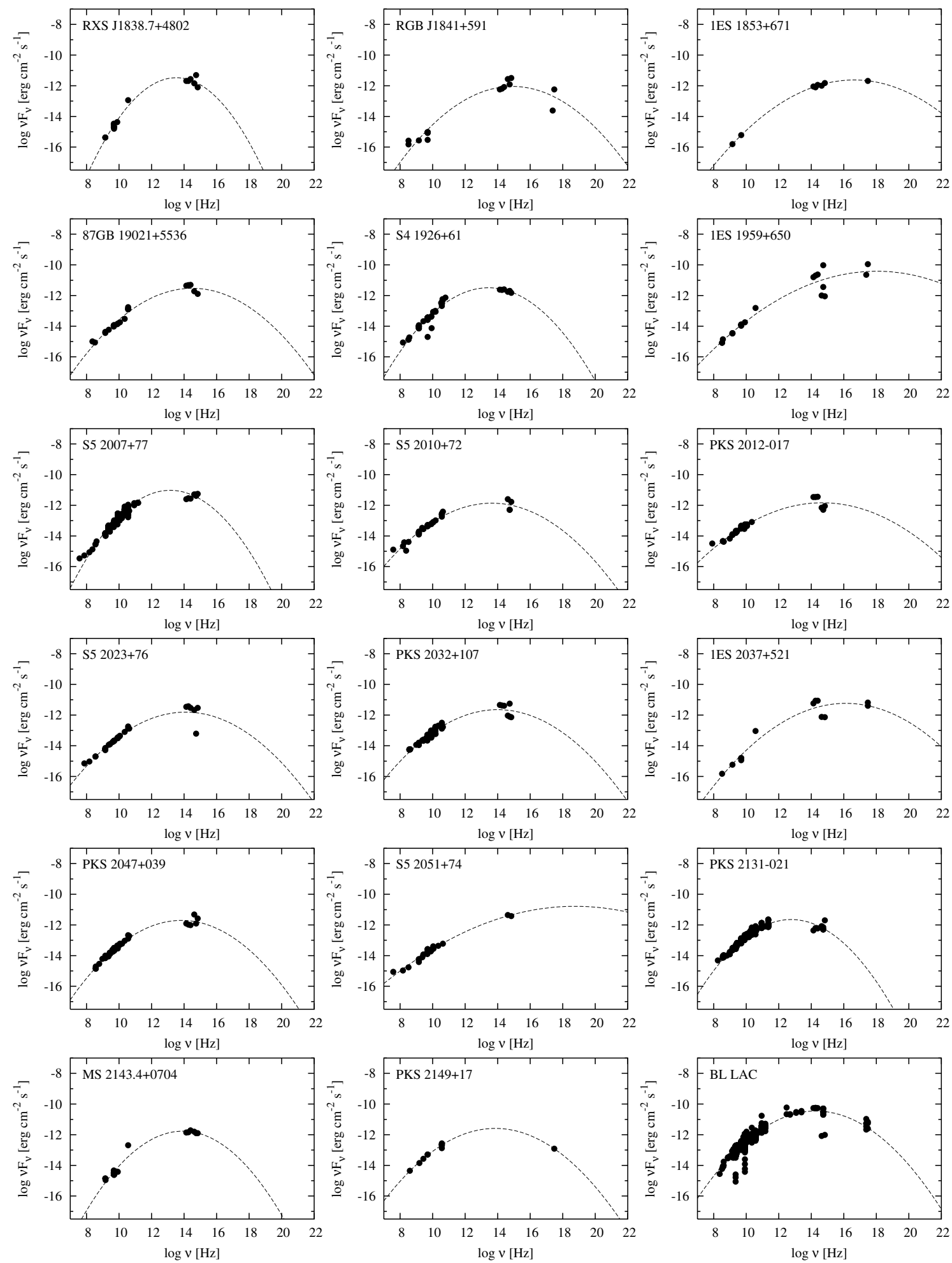

Fig. 13. continued. 
E. Nieppola et al.: SEDs of BL Lacertae Objects, Online Material p 26
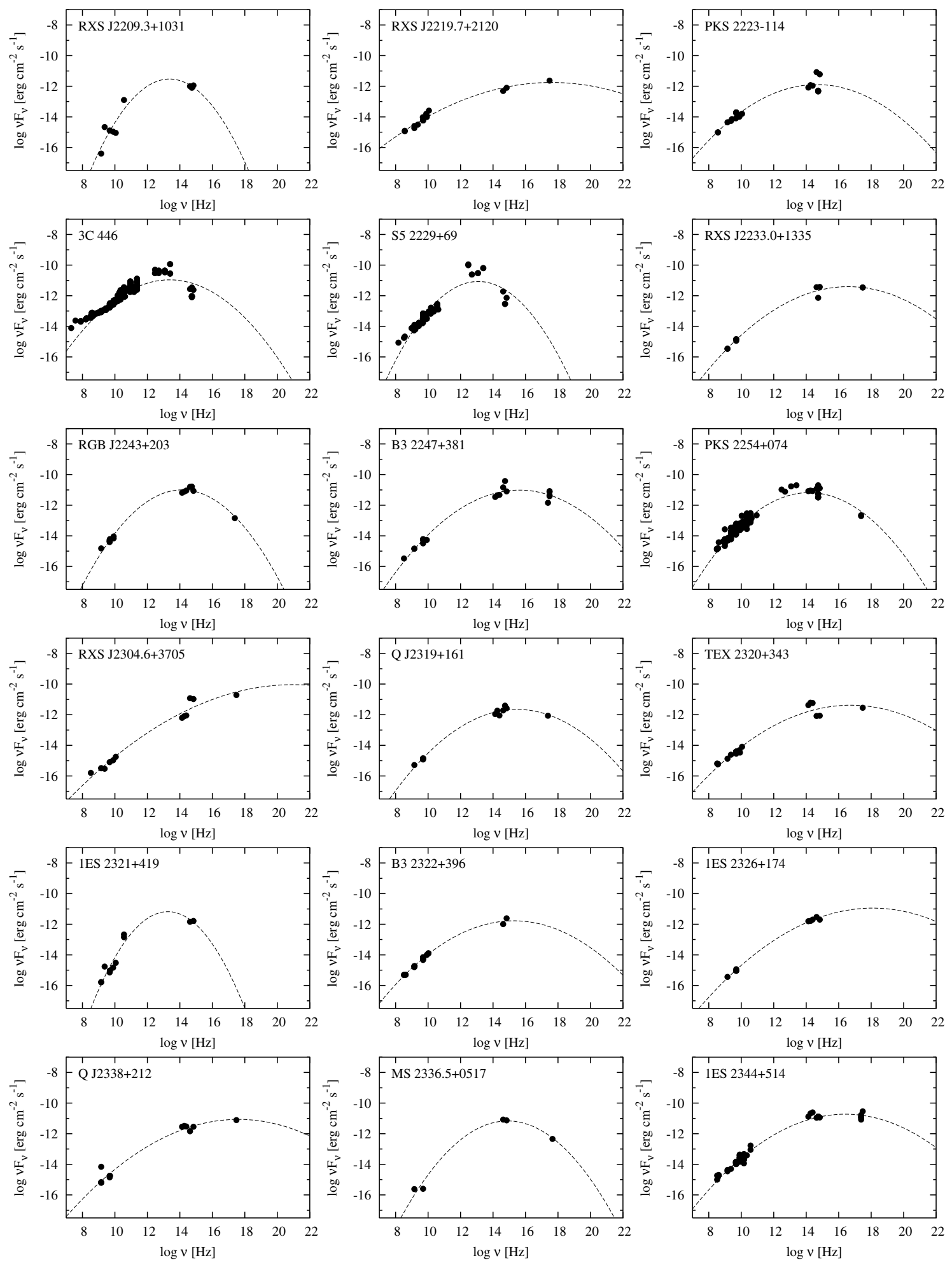

Fig. 13. continued. 
E. Nieppola et al.: SEDs of BL Lacertae Objects, Online Material p 27
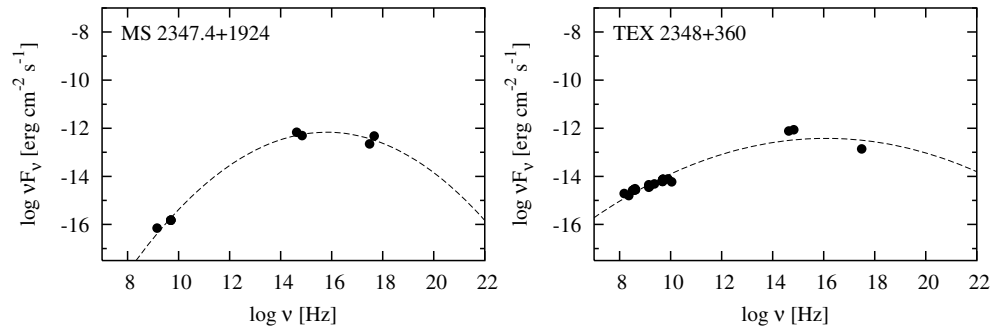

Fig. 13. continued. 